bioRxiv preprint doi: https://doi.org/10.1101/376806; this version posted July 25, 2018. The copyright holder for this preprint (which was not certified by peer review) is the author/funder. All rights reserved. No reuse allowed without permission.

\title{
Yeast Chd1p remodels nucleosomes with unique DNA unwrapping and translocation dynamics
}

\author{
Jaewon Kirk ${ }^{1 \dagger}$, Ju Yeon Lee ${ }^{1 \dagger}$, Yejin Lee ${ }^{2 \dagger}$, Soochul Shin ${ }^{1}$, Eunhye Lee ${ }^{2}$, Ji-Joon Song ${ }^{2 *}$, and \\ Sungchul Hohng ${ }^{1 *}$
}

${ }^{1}$ Department of Physics and Astronomy, Institute of Applied Physics, Seoul National University, Seoul 08826, Republic of Korea.

${ }^{2}$ Department of Biological Sciences, KI of the BioCentury, Korea Advanced Institute of Science and Technology (KAIST), Daejeon 34141, Republic of Korea.

*Correspondence should be addressed to songj@kaist.ac.kr (J. S.) or shohng@snu.ac.kr (S. H.)

$\dagger$ These authors contributed equally to this work.

\begin{abstract}
Chromodomain-helicase-DNA-binding protein 1 (CHD1) remodels chromatin by translocating nucleosomes along DNA, but its mechanism remains poorly understood. Here, we employ a single-molecule fluorescence approach to characterize nucleosome remodeling by yeast CHD1 (Chd1p). We show that Chd1p translocates nucleosomes in steps of multiple base pairs per ATP. ATP binding to Chd $1 p$ induces a transient unwrapping of the exit-side DNA, and facilitates nucleosome translocation. ATP hydrolysis induces nucleosome translocation, which is followed by the rewrapping upon the release of the hydrolyzed nucleotide. Multiple Chd1ps binding to a single nucleosome sequentially moves a histone octamer with a preference to the center of DNA fragments, suggesting a new mechanism for regularly spaced nucleosome generation by Chd1p. Our results reveal the unique mechanism by which Chd1p remodels nucleosomes.
\end{abstract}

One Sentence Summary: Yeast CHD1 uses one ATP to unwrap and translocate a nucleosome by multiple base pairs.

\section{Introduction}

Eukaryotic DNA is packaged hierarchically into a complex structure called chromatin.

Chromatin is made up of fundamental units called nucleosomes, which are composed of 146 base pairs of DNA wrapped around a histone octamer $(1,2)$. DNA wrapped around histone cores to form nucleosomes is much less accessible to DNA-binding regulatory proteins (3). Cells, therefore, use ATP-dependent chromatin remodelers to modulate DNA accessibility during processes like DNA transcription, replication, and repair (4-7). There are four major ATPdependent chromatin remodeler families: SWI/SNF, ISWI, CHD, and INO80/SWR1 (8). Most of these ATP-dependent chromatin remodelers form large multi-subunit complexes, each with a unique ATPase subunit that belongs to helicase superfamily 2 (5). In addition to this ATPase domain, each ATPase subunit contains several other distinct functional domains (9). For example, CHD family remodelers contain a chromo-domain that interacts with methyl-histone and/or DNA (5, 10-14). SWI/SNF family ATPases have C-terminal bromo domains that recognize acetylated histones $(15,16)$. The ATPase domain is thought to provide the mechanical 
force necessary for nucleosome remodeling, while the other domains in the ATPase subunit and other subunits in the complexes are thought to regulate the ways this mechanical force is applied in distinct situations and mechanisms. The mechanisms by which some of the chromatin remodelers - including ISWI, RSC, ACF, and CHD1 - alter nucleosome structure have been explored with single-molecule technologies (17-33). In contrast to other chromatin remodelers for which the precise molecular mechanisms of nucleosome remodeling are relatively well studied, it remains still unclear how CHD1 remodels a nucleosome. Here, using single-molecule FRET (Fluorescence Resonance Energy Transfer) $(34,35)$, we unveil a novel mechanism by which yeast CHD1 (Chd1p) remodels nucleosomes.

\section{RESULT}

\section{Yeast Chd1p remodels nucleosomes with multiple base pair kinetic steps}

ISWI and SWI/SNF family chromatin remodelers translocate nucleosomes in 1-2-bp steps (18, 19). A structural study on Chd1p also proposed 1-bp translocation mechanism for the CHDfamily remodeler (36). We examined using single-molecule FRET (17) whether the same mechanism of 1-bp translocation is valid for Chd1p. We prepared nucleosomes using Widom 601 DNA sequence (37), labeled with Cy5 at the end of the DNA on the exit side, and Cy3 on $\mathrm{H} 2 \mathrm{~A}$ of the histone octamer. The $\mathrm{Cy} 5$ labeling site was selected to facilitate high levels of FRET before remodeling (Fig. S1A). We verified that fluorophore-labeling does not affect the remodeling activity of Chd1p (Fig. S1B). We then immobilized the nucleosomes on a polymercoated quartz surface using the streptavidin-biotin interaction. We observed three peaks in the distribution of the FRET signal from the surface-immobilized nucleosomes, each corresponding to three different labeled species (Fig. S2). Of these three species, we used only the one with the highest levels of FRET in which Cy3 is attached to a position proximal to the exit side. This meant we were able to use FRET decrease to monitor the translocation of DNA at the exit side. After incubating the surface-immobilized nucleosomes with Chd1p, we added ATP to start nucleosome remodeling (Fig. 1A). Remodeling by Chd1p produced a stepwise reduction in the FRET signal (P1 and P2 in Fig. 1B), which suggests Chd1p remodels nucleosomes in a unidirectional manner. As previously observed for Chd1p as well as ACF $(17,21)$, we observed a recovery of FRET efficiency to its original value after an initial remodeling step (29\% of remodeling events, Fig. S3A), which suggests that the nucleosome repositioning process can be reversed. The nature of this backward translocation by Chd1p is not pursued here.

We were interested to observe two distinct populations in the FRET histograms after the first remodeling event (P1, Fig. 1C). Using FRET data calibrated with varying exit linker lengths (Fig. S4), we estimated that these two FRET populations correspond to small (4.4 \pm 1.1 -bp) and large (7.4 $\pm 1.4-\mathrm{bp})$ translocations. The second remodeling step (P2) also exhibited two FRET peaks, probably also corresponding to the small and large translocations (Fig. 1D). These occurred independently of the first remodeling step size (Fig. 1D), indicating that the step size is stochastically determined.

\section{Chd1p uses one ATP for each kinetic remodeling step}

Other chromatin remodelers translocate nucleosomes with a 1 or 2-bp step per ATP molecule (ISWI, RSC) $(18,19)$. Although ISWI translocates nucleosomes with 3 or 7-bp kinetic steps, 
these kinetic steps comprise multiple 1-bp substeps (18). We, therefore, decided to examine whether the kinetic steps of Chd1p may also be composed of 1-bp substeps. First, we tried to observe the substeps by injecting the mixture of minimal ATP and saturating ATP $\gamma$ S (18). In contrast to ISWI chromatin remodelers which exhibited 1-bp substeps, we could not detect any substep (Fig. 2A), suggesting that the two-small and large-kinetic steps observed in figure 1 are fundamental. However, this observation does not answer the question of how many ATPs are used for each fundamental remodeling step because multiple Chd1p may cooperatively function during the nucleosome remodeling. Chd1p is known to function as a monomer (38), but we found that multiple Chd1p can bind to a nucleosome in our experimental condition (Fig. S5). To observe the remodeling by monomeric Chd1p, we immobilized Chd1p using streptavidin-biotin interaction, and added a nucleosome labeled with a FRET pair (21). In this case Chd1p exhibited

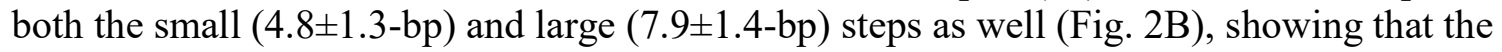
kinetic translocation steps of multiple base pairs are not due to multimerization of Chd1p. To confirm that single ATP is used for both the small, and large remodeling steps, we preloaded nucleosome-Chd1p complexes with ATP. After washing free ATP from the reaction chamber, we added $\mathrm{Mg}^{2+}$ to start remodeling and then measured changes in FRET. Before the injection of $\mathrm{Mg}^{2+}$, free ATP was thoroughly washed out from the reaction chamber. Considering the efficiency of the buffer exchange system used (Fig. S6-8), it can be safely assumed that only prebound-ATP molecules could be used for nucleosome remodeling. Under this condition, the FRET histogram after the first remodeling step also showed two peaks corresponding to the small and large remodeling steps regardless of whether Chd1p or a nucleosome is immobilized (Figure 2C-D). As expected from a monomeric Chd1p, when Chd1p was immobilized, only single remodeling steps were observed (Fig. 2C). Surprisingly, however, when a nucleosome was immobilized, we could observe multiple remodeling steps (Fig. 2D), indicating that multiple Chd1ps binding to a single nucleosome can sequentially and unidirectionally remodel a nucleosome. In the case of figure 2D, the reversal of the nucleosome remodeling (Fig. S3B) was also observed with $33 \%$ probability.

\section{ATP binding to Chd1p induces stochastic DNA unwrapping on the exit side}

The FRET change during Chdlp-mediated nucleosome remodeling exhibited a characteristic, but not monotonic pattern; we consistently observed down-spikes in the FRET signal just before each translocation event (Fig. 1B). This suggests that the nucleosome structure is being altered right before nucleosome translocation. To better characterize the nature of these down-spikes, we performed similar remodeling experiments under different labeling schemes. First, we looked for Chd1p-induced conformational changes in the histone octamers using Cy3-H2A and Cy5-H4. In this case, we did not observe any change in the FRET signal (Fig. S9A), indicating that Chd1p does not alter the histone octamer conformation. Next, to determine whether the down-spikes can be attributed to the dye labeling at specific positions on the histones, we moved $\mathrm{Cy} 3$ from $\mathrm{H} 2 \mathrm{~A}$ to H4 (Method). This had no effect on the down-spikes (Fig. S9B). The down spikes coupled with a nucleosome remodeling were consistently observed when the dye labeling position on DNA was moved around the exit side (Fig. S9C-F). We, therefore, conclude that the FRET down-spikes are caused by substantial unwrapping of the DNA on the exit side prior to nucleosome translocation by Chd1p. Recent structural studies based on cryo-EM revealed DNA unwrapping in the presence of $\mathrm{ADP}_{-} \mathrm{BeF}_{3}(11,20,36)$. As DNA unwrapping occurs before DNA translocation, which requires ATP hydrolysis, we asked whether ATP binding itself is involved 
in the unwrapping that occurs on the exit side. We performed a double flow experiment by adding ATP $\gamma$ S followed by a step to wash the excess ATP $\gamma \mathrm{S}$ from the reaction chamber (Fig. 3A). When we added ATP $\gamma$ S, we observed down-spikes without changes in the FRET signal that corresponded to nucleosome translocation. This suggests DNA unwrapping occurs without ATP hydrolysis or nucleosome translocation. In some cases, we still observed the down-spikes for a while even after removal of ATP $\gamma$ S (Fig. 3A), suggesting that the already bound ATP $\gamma$ S can induce unwrapping of exit side DNA several times. This retention of ATP analog by Chd1p explains why the $\mathrm{Mg}^{2+}$ flow assay described in figure 2C-D works. Consistently with the cryoEM studies (11), we found that ADP-BeF 3 induced stable DNA unwrapping (Fig. S10A). Interestingly, other ATP analogs, AMP-PNP and $\mathrm{ADP}-\mathrm{AlF}_{4}$, did not induce any DNA unwrapping (Fig S10B-C). To further investigate the relation between the binding of ATP $\gamma$ S and DNA unwrapping, we performed a kinetic analysis of the time delay between ATP $\gamma$ S injection and the first down-spike $\left(t_{i}\right)$, the down-spike dwell time $\left(t_{d}\right)$, and the high FRET dwell time after the first down-spike $\left(t_{h}\right)$ at varying ATP $\gamma \mathrm{S}$ concentrations (Figure 3B-D). The fact that all three of these kinetic parameters $\left(t_{i}, t_{d}\right.$, and $\left.t_{h}\right)$ can be nicely fitted to single exponential functions suggests each kinetic step has a single rate-limiting step. The ATP $\gamma \mathrm{S}$ titration data showed that $t_{i}$ and $t_{h}$ depend on ATP $\gamma$ S concentration (Fig. 3E, Fig. S11A-B), whereas $t_{d}$ seems to be independent of ATP $\gamma$ S concentration (Fig. 3F, Fig. S11C). Consistent with the observation that single ATP $\gamma \mathrm{S}$ binding can induce multiple DNA unwrapping (Fig. $3 \mathrm{~A}$ ), $\mathrm{t}_{\mathrm{h}}$ is shorter than $\mathrm{t}_{\mathrm{i}}$ at low ATP $\gamma \mathrm{S}$ concentrations. Thus, we conclude that DNA on the exit side is stochastically unwrapped by Chd1p while it is bound to ATP.

\section{Translocated DNA on the exit side is rewrapped after the dissociation of phosphate and ADP}

To further characterize how ATP is used during Chd1p-mediated nucleosome remodeling, we analyzed at varying ATP concentrations the delay between ATP injection and the first downspike $\left(t_{i}\right)$, the dwell time for down-spikes uncoupled to nucleosome translocation $\left(t_{d u}\right)$, and the dwell time for down-spikes coupled to nucleosome translocation $\left(t_{d c}\right)$ as defined in figure 4A; we noticed FRET down-spikes frequently occurring just before remodeling, but remodeling did not necessarily always follow a down-spike, and therefore distinguished down-spikes coupled to translocation from down-spikes uncoupled to translocation. The $t_{i}$ data are well-fitted to a single exponential function (Fig. 4B). Interestingly, the distributions of $t_{d u}$ and $t_{d c}$ were clearly different from one another (Fig. 4C-D). The $t_{\mathrm{du}}$ data are reasonably well-fitted to a single-exponential distribution, while the $t_{d c}$ data are well-fitted to a gamma distribution. The fact that the $t_{d c}$ data cannot be fitted to a single exponential distribution suggests multiple steps are involved in the kinetics of DNA rewrapping when it is coupled to nucleosome translocation. In addition, while $t_{i}$ depended on ATP concentration (Fig. 4E, Fig. S12A), the $t_{d u}$ and $t_{d c}$ data are independent of ATP concentration (Fig. 4F, Fig. S12B-C), supporting the conclusion that these events occur in the ATP-bound state. To determine whether the status of the hydrolyzed nucleotide after nucleosome translocation affects the duration of DNA unwrapping, we performed vanadate (39) and ADP titration experiments. At high vanadate or ADP concentrations, we observed increases in $t_{d c}$, whereas $t_{d u}$ remained unaffected (Fig. 4G-H, Fig. S13). These data suggest that the DNA unwrapping state coupled to nucleosome translocation is maintained as long as the hydrolyzed nucleotide remains bound to Chd $1 \mathrm{p}$ probably after nucleosome translocation. Then, upon phosphate and ADP dissociation, the translocated DNA is rewrapped. We confirmed that the 
bioRxiv preprint doi: https://doi.org/10.1101/376806; this version posted July 25, 2018. The copyright holder for this preprint (which was not certified by peer review) is the author/funder. All rights reserved. No reuse allowed without permission.

pause between remodeling events ( $t_{p 1}$ in Fig. 4A) depended on ATP concentration (Fig. S14), supporting the expectation that a new ATP hydrolysis cycle is required for each remodeling step.

\section{Translocation occurs simultaneously on both the entry and exit sides}

Cryo-EM studies revealed DNA unwrapping in the presence of $\operatorname{ADP}_{-B_{3}}(11,20,36)$. Singlemolecule FRET study showed that remodeling by ISWI proceeds with a time delay between the entry and exit sides (18). We therefore asked whether DNA unwrapping on the entry side and a time delay similar to the case of ISWI-mediated remodeling exist with Chd $1 \mathrm{p}$-mediated remodeling using nucleosomes labeled at the entry side (Fig. S1A). In contrast to what we observed with labeling at the exit side, we observed frequent FRET down-spikes even when Chd1p alone was injected without ATP (Fig. 5A). When we added ATP to initiate nucleosome remodeling, as expected from the results of the exit side remodeling (Fig. 1-2), we observed remodeling events corresponding to small and large remodeling steps (Fig. S15A-B). Interestingly, repetitive down-spikes were observed before ATP injection only in some remodeled molecules (top, Fig. 5B), but not in others (bottom, Fig. 5B). We do not currently understand the origin of the heterogeneity. When we did observe the down-spikes before remodeling, they became less frequent upon ATP injection (top, Fig. 5B; Fig. S15C). We also tested the degree to which the down-spikes were coupled to the translocation events and found that coupling on the entry side is significantly less than the exit side ( $22 \%$ vs. $77 \%$, Fig. $5 \mathrm{C})$. In case of the exit side remodeling, the coupling efficiency of the down spike and remodeling was apparently higher than the probability for a down-spike to occur simultaneously with nucleosome remodeling by chance, but the difference between them was not significant in the case of entry side remodeling (Fig. 5C). Consistently with the cryo-EM studies $(20,36)$ and previous singlemolecule FRET study (20), DNA on the entry side was unwrapped in the presence of various ATP analogs (Fig. S16).

To further study any possible role of DNA unwrapping on the entry side, we then performed a kinetic analysis of $t_{i}, t_{d u}$, and $t_{d c}$ for the entry side. $t_{i}$ was clearly shorter on the entry side than the exit side (Fig. S15D), indicating that the unwrapping events of DNA on the entry and exit sides are unsynchronized. In contrast to what we observed with the exit side, the distributions of the $t_{d u}$ and $t_{d c}$ data for the entry side were similar (Fig. 5D). In contrast to the marked difference in the DNA unwrapping dynamics on the entry and exit sides, the time delay between ATP injection and the first translocation event ( $t_{r}$ in Fig. 5B) was similar for both the exit and entry sides (Fig. 5E). This indicates that nucleosome translocation occurs simultaneously on both the exit and entry sides. Considering all these observations, DNA unwrapping on the entry side is not strongly coupled with nucleosome translocation, and the role of DNA unwrapping on the entry side in Chd1p-mediated nucleosome remodeling is not clear.

\section{DNA unwrapping on the exit side dictates the remodeling speed}

Our data suggest DNA unwrapping on the exit side is a critical step for a nucleosome remodeling by Chd1p. It is shown that DNA flexibility is a determining factor for DNA unwrapping/wrapping dynamics (40). The nucleosome remodeling by Chdlp is strongly affected by DNA sequences (41). We therefore studied the correlation between DNA unwrapping on the exit side and a nucleosome remodeling by Chd1p using a series of DNA sequences with 
variation on the exit side (Fig. S17). $t_{i}$ and $t_{r}$ sensitively depended on DNA sequences (Fig. 6AB) with a strong correlation between them (Pearson's r: 0.973, Fig. 6C). These data implicate that both the nucleosome remodeling efficiency and kinetics may be controlled by DNA sequences.

\section{DISCUSSION}

Recent studies commonly suggested that superfamily 2 (SF2) helicases including several chromatin remodelers translocate on DNA with 1-bp step in general $(18,19,42,43)$. Based on structural information but without direct evidence, Chd1p was also assumed to translocate a nucleosome with 1-bp step (36). Surprisingly, however, we revealed that Chd1 p translocates nucleosomes in multiple base pair steps per one hydrolyzed molecule of ATP. As the ISWI and RSC remodelers were found to translocate nucleosomes in 1-2-bp steps $(18,19)$, larger translocation step sizes seem to be a defining characteristic of Chd1p.

Several static cryo-EM Chd1p-nucleosome structures revealed DNA detachment $(11,20,36)$, but it has remained unclear exactly what role DNA unwrapping plays during nucleosome remodeling. We revealed that DNA unwrapping on the exit side is strongly coupled with, and accelerates DNA translocation along a histone octamer. Although further studies are required, it is reasonably conceivable that DNA unwrapping on the exit side facilitates DNA translocation by weakening DNA-histone interactions. Although other groups have published single-molecule FRET studies of other chromatin remodelers including ACF, ISWI, and RSC (17-19), dynamic DNA unwrapping on the exit side strongly coupled with DNA translocation has never been reported. DNA unwrapping may represent another unique characteristic of Chd1p. Several previous studies suggested the existence of DNA looping during the remodeling process (44-46), but the DNA unwrapping we observed does not seem to be related to the DNA looping. In the looping model, DNA unwrapping coupled with remodeling should occur on the entry side not the exit side.

It has always been a fascinating question how molecular motors couple each step of ATP hydrolysis to their function. We revealed that ATP binding to Chd1p induces DNA unwrapping on the exit side of nucleosome, that the step of ATP hydrolysis is coupled to DNA translocation, and that the phosphate and hydrolyzed nucleotide must be released before DNA rewrapping can occur following nucleosome translocation (Fig. 6D). Therefore, it seems that the individual steps of the ATP hydrolysis cycle (i.e., ATP binding, ATP hydrolysis, and release of ADP and a phosphate) are each coupled to a distinct step of the nucleosome remodeling process (i.e., DNA unwrapping, DNA translocation, and DNA rewrapping).

It is known that Chd1p generates regularly spaced nucleosomal arrays in vivo (47), and, consistently with the observation, moves histone octamers to the center of short DNA fragments in vitro (48-51), but its exact mechanism remains unknown. We revealed that multiple Chd1ps binding to a single nucleosome sequentially move a histone octamer with a preference to the center of DNA fragments (Fig. 6E). Such a demanding task of switching Chd1ps acting on a single nucleosome can be achieved only if the interaction of Chd1p and a nucleosome is highly dynamic (21). Further single-molecule FRET studies with labeled Chd1p will elucidate the dynamic nature of the Chd1p-nucleosome interactions. 
The results of several studies have suggested the different families of ATP-dependent chromatin remodelers each use a distinct nucleosome remodeling mechanism (52). This seems consistent with the presence of distinct protein domains in the ATPase subunits of each family and with the presence of distinct subunits in each remodeler complex. Consistently with this expectation, our single-molecule FRET analysis of the yeast CHD1 ATP-dependent chromatin remodeler revealed that the mechanism by which Chd1p remodels nucleosomes is distinct from those of remodelers from the ISWI and SWI/SNF families. It would be interesting to examine whether other remodelers in CHD family have similar properties to Chd1p. Considering that Chd1p, ISWI, and SWI/SNF are all members of the SF2 superfamily (17-19), it remains unclear exactly what about Chd1p makes it function in this way. To address the exact mechanism of these characteristic features of Chd $1 \mathrm{p}$ might require a series of high-resolution structures in different ATP hydrolysis states.

\section{References and Notes:}

1. R. D. Kornberg, Chromatin structure: a repeating unit of histones and DNA. Science 184, 868-871 (1974).

2. K. Luger, A. W. Mäder, R. K. Richmond, D. F. Sargent, T. J. Richmond, Crystal structure of the nucleosome core particle at $2.8 \AA$ resolution. Nature 389, 251 (1997).

3. R. D. Kornberg, Y. Lorch, Twenty-five years of the nucleosome, fundamental particle of the eukaryote chromosome. Cell 98, 285-294 (1999).

4. B. Li, M. Carey, J. L. Workman, The role of chromatin during transcription. Cell 128, 707-719 (2007).

5. C. R. Clapier, B. R. Cairns, The biology of chromatin remodeling complexes. Annual review of biochemistry 78, 273-304 (2009).

6. G. D. Bowman, Mechanisms of ATP-dependent nucleosome sliding. Current opinion in structural biology 20, 73-81 (2010).

7. C. R. Clapier, B. R. Cairns, Regulation of ISWI involves inhibitory modules antagonized by nucleosomal epitopes. Nature 492, 280 (2012).

8. A. Flaus, D. M. Martin, G. J. Barton, T. Owen-Hughes, Identification of multiple distinct Snf2 subfamilies with conserved structural motifs. Nucleic acids research 34, 2887-2905 (2006).

9. M. R. Singleton, M. S. Dillingham, D. B. Wigley, Structure and mechanism of helicases and nucleic acid translocases. Annu. Rev. Biochem. 76, 23-50 (2007).

10. K. Bouazoune et al., The dMi-2 chromodomains are DNA binding modules important for ATP-dependent nucleosome mobilization. The EMBO journal 21, 2430-2440 (2002).

11. I. M. Nodelman et al., Interdomain communication of the Chd1 chromatin remodeler across the DNA gyres of the nucleosome. Molecular cell 65, 447-459. e446 (2017).

12. J. F. Flanagan et al., Double chromodomains cooperate to recognize the methylated histone H3 tail. Nature 438, 1181 (2005).

13. R. J. Sims et al., Human but not yeast CHD1 binds directly and selectively to histone H3 methylated at lysine 4 via its tandem chromodomains. Journal of Biological Chemistry 280, 41789-41792 (2005).

14. M. G. Pray-Grant, J. A. Daniel, D. Schieltz, J. R. Yates III, P. A. Grant, Chd1 chromodomain links histone H3 methylation with SAGA-and SLIK-dependent acetylation. Nature 433, 434 (2005).

15. A. H. Hassan et al., Function and selectivity of bromodomains in anchoring chromatin-modifying complexes to promoter nucleosomes. Cell 111, 369-379 (2002).

16. M. Kasten et al., Tandem bromodomains in the chromatin remodeler RSC recognize acetylated histone $\mathrm{H} 3$ Lys 14. The EMBO journal 23, 1348-1359 (2004).

17. T. R. Blosser, J. G. Yang, M. D. Stone, G. J. Narlikar, X. Zhuang, Dynamics of nucleosome remodelling by individual ACF complexes. Nature 462, 1022 (2009).

18. S. Deindl et al., ISWI remodelers slide nucleosomes with coordinated multi-base-pair entry steps and single-base-pair exit steps. Cell 152, 442-452 (2013).

19. B. T. Harada et al., Stepwise nucleosome translocation by RSC remodeling complexes. Elife 5, (2016).

20. R. Sundaramoorthy et al., Structural reorganization of the chromatin remodeling enzyme Chd1 upon engagement with nucleosomes. Elife 6, (2017). 
bioRxiv preprint doi: https://doi.org/10.1101/376806; this version posted July 25, 2018. The copyright holder for this preprint (which was not certified by peer review) is the author/funder. All rights reserved. No reuse allowed without permission.

Submitted Manuscript: Confidential

\section{MAAAS}

21. Y. Qiu et al., The Chd1 Chromatin Remodeler Shifts Nucleosomal DNA Bidirectionally as a Monomer. Molecular cell 68, 76-88. e76 (2017).

22. B. D. Brower-Toland et al., Mechanical disruption of individual nucleosomes reveals a reversible multistage release of DNA. Proceedings of the National Academy of Sciences 99, 1960-1965 (2002).

23. A. H. Mack, D. J. Schlingman, R. P. Ilagan, L. Regan, S. G. Mochrie, Kinetics and thermodynamics of phenotype: unwinding and rewinding the nucleosome. Journal of molecular biology 423, 687-701 (2012).

24. S. Mihardja, A. J. Spakowitz, Y. Zhang, C. Bustamante, Effect of force on mononucleosomal dynamics. Proceedings of the National Academy of Sciences 103, 15871-15876 (2006).

25. Y. Zhang et al., DNA translocation and loop formation mechanism of chromatin remodeling by SWI/SNF and RSC. Molecular cell 24, 559-568 (2006).

26. A. Gansen et al., Nucleosome disassembly intermediates characterized by single-molecule FRET. Proceedings of the National Academy of Sciences 106, 15308-15313 (2009).

27. M. A. Hall et al., High-resolution dynamic mapping of histone-DNA interactions in a nucleosome. Nature Structural and Molecular Biology 16, 124 (2009).

28. C. Hodges, L. Bintu, L. Lubkowska, M. Kashlev, C. Bustamante, Nucleosomal fluctuations govern the transcription dynamics of RNA polymerase II. Science 325, 626-628 (2009).

29. M. Kruithof, J. van Noort, Hidden Markov analysis of nucleosome unwrapping under force. Biophysical journal 96, 3708-3715 (2009).

30. M. Y. Sheinin, M. Li, M. Soltani, K. Luger, M. D. Wang, Torque modulates nucleosome stability and facilitates H2A/H2B dimer loss. Nature communications 4, 2579 (2013).

31. A. Shundrovsky, C. L. Smith, J. T. Lis, C. L. Peterson, M. D. Wang, Probing SWI/SNF remodeling of the nucleosome by unzipping single DNA molecules. Nature Structural and Molecular Biology 13, 549 (2006).

32. B. Sudhanshu et al., Tension-dependent structural deformation alters single-molecule transition kinetics. Proceedings of the National Academy of Sciences 108, 1885-1890 (2011).

33. J. Y. Lee et al., NAP1L1 accelerates activation and decreases pausing to enhance nucleosome remodeling by CSB. Nucleic acids research 45, 4696-4707 (2017).

34. T. Ha et al., Probing the interaction between two single molecules: fluorescence resonance energy transfer between a single donor and a single acceptor. Proceedings of the National Academy of Sciences 93, 62646268 (1996).

35. A. N. Kapanidis et al., Alternating-laser excitation of single molecules. Accounts of chemical research 38 , 523-533 (2005).

36. L. Farnung, S. M. Vos, C. Wigge, P. Cramer, Nucleosome-Chd1 structure and implications for chromatin remodelling. Nature 550, 539 (2017).

37. P. Lowary, J. Widom, New DNA sequence rules for high affinity binding to histone octamer and sequencedirected nucleosome positioning1. Journal of molecular biology 276, 19-42 (1998).

38. H. G. Tran, D. J. Steger, V. R. Iyer, A. D. Johnson, The chromo domain protein Chd1p from budding yeast is an ATP-dependent chromatin-modifying factor. The EMBO journal 19, 2323-2331 (2000).

39. D. R. Davies, W. G. Hol, The power of vanadate in crystallographic investigations of phosphoryl transfer enzymes. FEBS letters 577, 315-321 (2004).

40. T. T. Ngo, Q. Zhang, R. Zhou, J. G. Yodh, T. Ha, Asymmetric unwrapping of nucleosomes under tension directed by DNA local flexibility. Cell 160, 1135-1144 (2015).

41. J. Winger, G. D. Bowman, The Sequence of Nucleosomal DNA Modulates Sliding by the Chd1 Chromatin Remodeler. Journal of molecular biology 429, 808-822 (2017).

42. S. Myong, M. M. Bruno, A. M. Pyle, T. Ha, Spring-loaded mechanism of DNA unwinding by hepatitis C virus NS3 helicase. Science 317, 513-516 (2007).

43. W. Cheng, S. G. Arunajadai, J. R. Moffitt, I. Tinoco, C. Bustamante, Single-base pair unwinding and asynchronous RNA release by the hepatitis C virus NS3 helicase. Science 333, 1746-1749 (2011).

44. R. Strohner et al., A'loop recapture'mechanism for ACF-dependent nucleosome remodeling. Nature Structural and Molecular Biology 12, 683 (2005).

45. M. Zofall, J. Persinger, S. R. Kassabov, B. Bartholomew, Chromatin remodeling by ISW2 and SWI/SNF requires DNA translocation inside the nucleosome. Nature Structural and Molecular Biology 13, 339 (2006).

46. G. Lia et al., Direct observation of DNA distortion by the RSC complex. Molecular cell 21, 417-425 (2006).

47. A. Lusser, D. L. Urwin, J. T. Kadonaga, Distinct activities of CHD1 and ACF in ATP-dependent chromatin assembly. Nature Structural and Molecular Biology 12, 160 (2005). 
48. C. Stockdale, A. Flaus, H. Ferreira, T. Owen-Hughes, Analysis of nucleosome repositioning by yeast ISWI and Chd1 chromatin remodeling complexes. Journal of Biological Chemistry 281, 16279-16288 (2006).

49. G. Hauk, J. N. McKnight, I. M. Nodelman, G. D. Bowman, The chromodomains of the Chd1 chromatin remodeler regulate DNA access to the ATPase motor. Molecular cell 39, 711-723 (2010).

50. A. Patel, J. N. McKnight, P. Genzor, G. D. Bowman, Identification of residues in chromodomain helicase DNA-binding protein 1 (Chd1) required for coupling ATP hydrolysis to nucleosome sliding. Journal of Biological Chemistry 286, 43984-43993 (2011).

51. I. M. Nodelman, G. D. Bowman, Nucleosome sliding by Chd1 does not require rigid coupling between DNA-binding and ATPase domains. EMBO reports 14, 1098-1103 (2013).

52. C. R. Clapier, J. Iwasa, B. R. Cairns, C. L. Peterson, Mechanisms of action and regulation of ATPdependent chromatin-remodelling complexes. Nature Reviews Molecular Cell Biology 18, 407 (2017).

\section{Acknowledgments}

This work was supported by a Creative Research Initiative grant (Physical Genetics Laboratory, 2009-0081562) to S.H. and by grants (NRF-2016R1A2B3006293, NRF-2016K1A1A2912057) to J.S. from National Research Foundation of Korea.

\section{Data and materials availability}

All data needed to evaluate the conclusions in the paper are present in the paper and/or the Supplementary Materials. Additional data related to this paper may be requested from the authors.

\section{Supplementary Materials: In Separate File}

Materials and Methods

Figure S1. DNA sequences and remodeling activity of Chd1p on labelled nucleosomes.

Figure S2. FRET histogram of the exit-labeled nucleosome.

Figure S3. Bidirectional nucleosome remodeling by Chd1p.

Figure S4. Linear relation between FRET efficiency and the exit linker length.

Figure S5. The number of Chd1ps on a DNA substrate with a nucleosome.

Figures S6-8. Characterization of the buffer exchange efficiency of the flow system I-III.

Figure S9. FRET down spikes on the exit side under different labeling schemes.

Figure S10. FRET down spikes on the exit side in the presence of various ATP analogs.

Figure S11. ATP $\gamma \mathrm{S}$ titration of kinetic parameters of the exit side unwrapping.

Figure S12. ATP titration of kinetic parameters of the exit side remodeling.

Figure S13. ATP titration of $\mathrm{t}_{\mathrm{p} 1}$.

Figure S14. Effects of vanadate and ADP on remodeling kinetics.

Figure S15. Supplementary data to characterize the entry-side remodeling.

Figure S16. FRET down spikes on the entry side in the presence of various ATP analogs.

Figure S17. DNA sequences used to study the correlation between DNA unwrapping on the exit side and nucleosome remodeling. 
Fig 1. The kinetic step size of nucleosome remodeling by Chd1p. (A) Experimental scheme. Nucleosomes labeled with a FRET-pair (Cy3, green circle; Cy5, red circle) were immobilized on a microscope slide and incubated with Chd1p (180 nM). ATP was injected into the reaction chamber to initiate chromatin remodeling. (B) Representative fluorescence intensity (top, green for Cy3 and red for Cy5) and FRET (bottom) time traces during nucleosome remodeling by Chd1p. ATP was injected into the detection chamber at 5 seconds (solid line). This same color convention is used throughout the paper. (C) FRET histogram during the first translocation pause (P1). The histogram is fitted to two Gaussian functions. (D) FRET histograms during the second translocation pause (P2) after first translocations of a small step (left) or a large step (right). The histograms were fitted to two Gaussian functions.

Fig 2. Nucleosome remodeling step size per ATP. Representative fluorescent intensity and FRET time traces (left), and FRET histograms during the first translocation pause (p1, right) for various experimental conditions: (A) Multiple Chd1ps are binding to a surface-immobilized nucleosome, and nucleosome remodeling is initiated by injecting $50 \mu \mathrm{M} \mathrm{ATP}$ and $500 \mu \mathrm{M}$ $\mathrm{ATP} \gamma \mathrm{S}$; (B) A nucleosome is binding to a surface-immobilized monomeric Chd1, and nucleosome remodeling is initiated by injecting $1 \mathrm{mM}$ ATP; (C) A nucleosome is binding to a surface-immobilized monomeric Chd1. The nucleosome-Chd1 $\mathrm{p}$ complex is incubated with $100 \mu \mathrm{M}$ ATP and 10mM EDTA, and nucleosome remodeling is initiated by injecting $3 \mathrm{mM} \mathrm{Mg}^{2+}$. (D) Multiple Chd1ps are binding to a surface-immobilized nucleosome. The nucleosome-Chd1p complex is incubated with $100 \mu \mathrm{M}$ ATP and $10 \mathrm{mM}$ EDTA, and nucleosome remodeling is initiated by injecting $3 \mathrm{mM} \mathrm{Mg}^{2+}$. From the molecules that exhibited unidirectional translocation, percentages of one, two, and three remodeling events were $72.5 \%, 23.2 \%$, and $4.3 \%$, respectively. All FRET histograms are fitted to two Gaussian functions.

\section{Fig 3. Stochastic unwrapping of the exit side DNA by Chd1p with ATP $\gamma$ S. (A)}

Representative fluorescence and FRET time traces showing FRET down-spikes upon addition of the ATP analog ATP $\gamma \mathrm{S}(1 \mathrm{mM})$ at 30 seconds (solid line). In some molecules, the down-spikes remained for a while even after the removal of free ATP $\gamma \mathrm{S}$ at 50 seconds (solid line). Kinetic parameters: $\mathrm{t}_{\mathrm{i}}$, the delay between ATP $\gamma \mathrm{S}$ injection and the first down-spike; $\mathrm{t}_{\mathrm{h}}$, the dwell time between two adjacent down-spikes; $t_{d}$, the dwell time of the down-spike. (B-D) Histograms of $t_{i}$, $t_{h}, t_{d}$ at $1 \mathrm{mM} \mathrm{ATP} \gamma S$, and their single-exponential fits (red lines). (E) ATP $\gamma S$ titration of $t_{i}$ and $t_{h}$, the data were obtained by fitting the histograms to single-exponential functions (Figure S11AB). (F) ATP $\gamma \mathrm{S}$ titration of $t_{d}$. The data were obtained by fitting the histograms to singleexponential functions (Figure S11C).

Fig 4. Rewrapping of the exit side DNA after phosphate and ADP dissociation. (A) Representative fluorescence intensity (top) and FRET (bottom) time traces reporting the translocation of the nucleosome after ATP injection at 10 seconds. Kinetic parameters: $t_{i}$, the time delay between ATP injection and the first down-spike; $t_{d u}$, the dwell time of down-spikes uncoupled to translocation; $t_{d c}$, the dwell time of down-spikes coupled to translocation. (B) A histogram of $t_{i}$ at $1 \mathrm{mM} \mathrm{ATP}$ and its fit to a single-exponential function (red line). (C) A histogram of $t_{d u}$ at $1 \mathrm{mM}$ ATP and its fit to a single-exponential function (red line). (D) A histogram of $t_{d c}$ at $1 \mathrm{mM}$ ATP and its fit to a gamma distribution. (E) ATP titration of $t_{i}$. The data were obtained by fitting the histograms to single-exponential functions (Fig. S12A). (F) ATP titration of $t_{d c}$ (black) and $t_{d u}$ (red). $t_{d u}$ data were obtained by fitting the histograms to singleexponential functions (Figure $\mathrm{S} 12 \mathrm{~B}$ ). $\mathrm{t}_{\mathrm{dc}}$ is the mean $\pm \mathrm{SEM}$ of those histograms (Fig. S12C). (G) 
Vanadate titration of $t_{d c}$ (black) and $t_{d u}(r e d)$ at $1 \mathrm{mM} \mathrm{ATP.} t_{d c}$ is the mean \pm SEM of those histograms (Fig. S13C). $t_{d u}$ data were obtained by fitting histograms to single-exponential functions (Fig. S13D). (H) ADP titration of $t_{d c}$ (black) and $t_{d u}$ (red) at $100 \mu \mathrm{M} \mathrm{ATP.} t_{d c}$ is the mean \pm SEM of those histograms (Fig. S13E). $t_{\text {du }}$ data were obtained by fitting histograms to single-exponential functions (Fig. S13F).

Fig 5. Entry-side remodeling by Chd1p. (A) Representative fluorescence intensity and FRET time traces for the entry-side labelled nucleosome in the presence of Chd1p. Chd1p was added at 10 seconds (solid line). (B) Representative fluorescence intensity (top) and their corresponding FRET (bottom) time traces showing remodeling on the entry side. Two different behaviors were observed: (upper panel) molecules showing down-spikes before ATP injection (85\%) and (lower panel) molecules showing no down-spikes before ATP injection (15\%). Kinetic parameters: $t_{i}$, the delay between ATP injection and the first down-spike; $t_{d u}$, the dwell time of down-spikes uncoupled to nucleosome translocation; $t_{d c}$, the dwell time of down-spikes coupled to nucleosome translocation; $t_{r}$, the time between ATP injection and translocation. (C) The coupling efficiency of down-spikes with translocation on the exit $(N=237)$ and entry sides $(N=162)$. The data are shown as means \pm SEM of 3 independent experiments. (D) Histograms of $t_{d u}$ (black) and $t_{\mathrm{dc}}(\mathrm{red})$ of the entry-side labelled nucleosome, in presence of $1 \mathrm{mM}$ ATP. (E) Histograms of $\mathrm{t}_{\mathrm{r}}$ on the exit-side labelled nucleosome $(N=237)$ and entry-sides labelled nucleosome $(N=162)$, in presence of $1 \mathrm{mM}$ ATP.

Fig 6. Correlation between DNA unwrapping on the exit side and nucleosome remodeling. $t_{i}$ (A) of a series of nucleosomes with sequence variation on the exit side (Fig. S17), and their $t_{r}$ (B). The experiments were performed in the presence of $1 \mathrm{mM}$ ATP. (C) Correlation plot of $1 / \mathrm{t}_{\mathrm{r}}$ and $1 / t_{i}$. The correlation coefficient (Pearson's r) was 0.974. (D) A proposed model of nucleosome remodeling by Chd1p. (E) A proposed model of sequential and unidirectional nucleosome remodeling by multiple Chd1ps. 


\section{Science Submitted Manuscript: Confidential}

\section{DIAAAS}

Figure 1

A

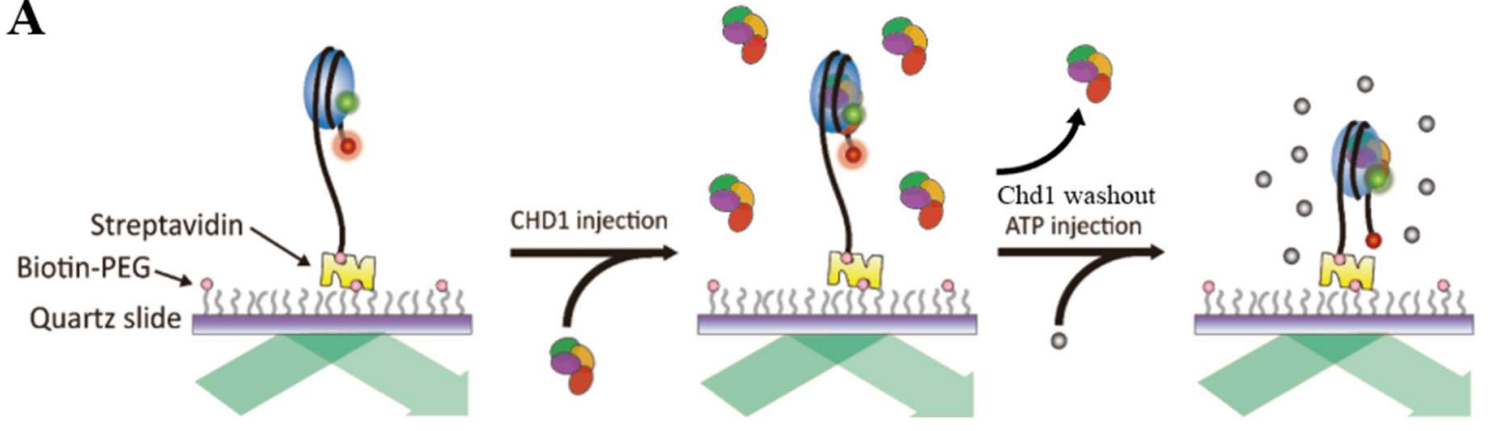

B

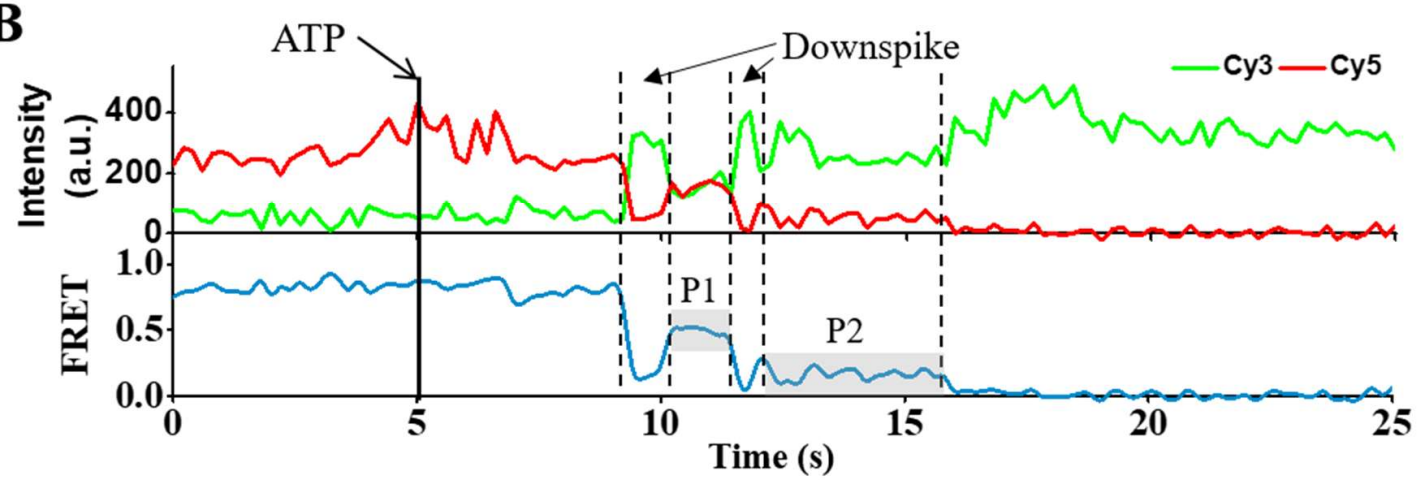

C

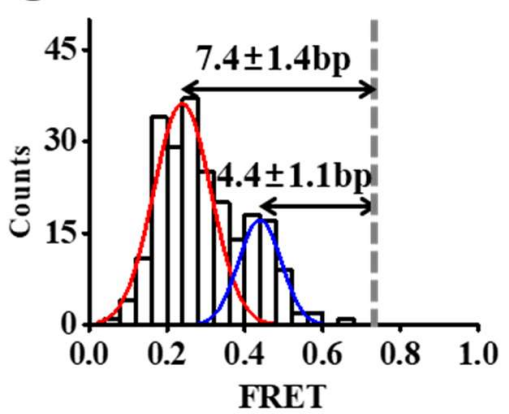

D
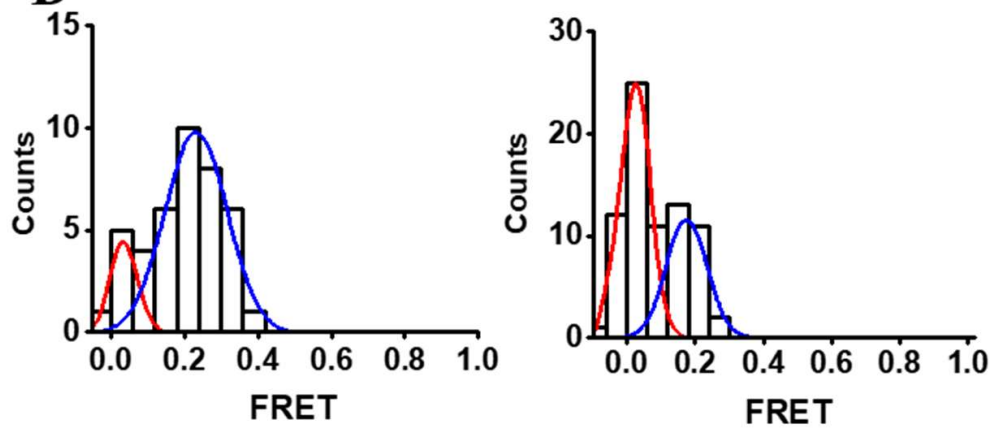


\section{Science}

\section{MAAAS}

Figure 2
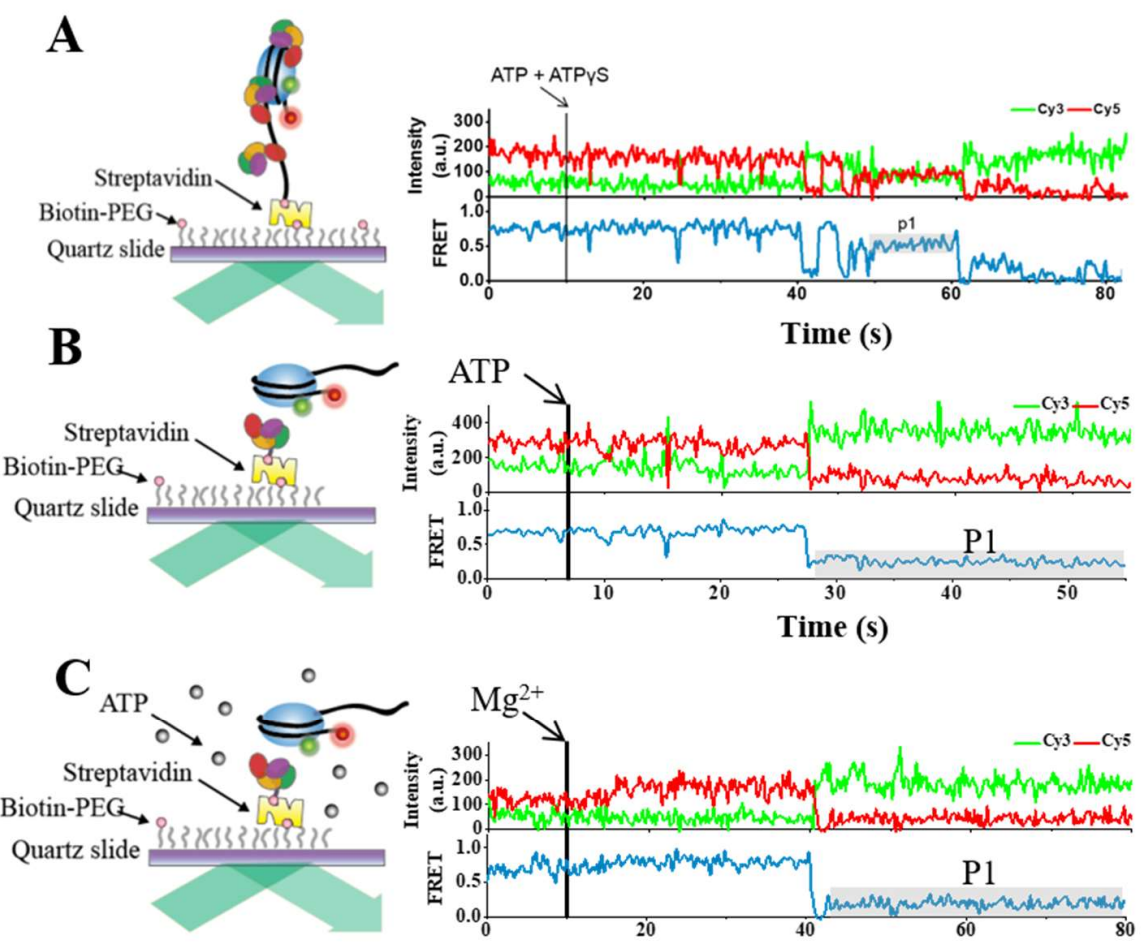

D
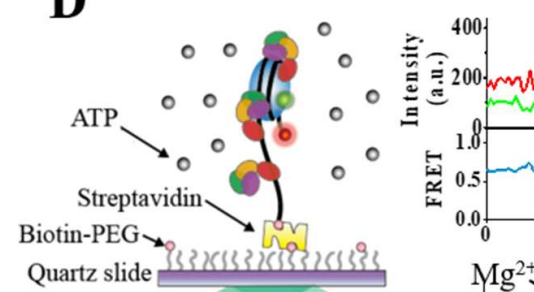

穿

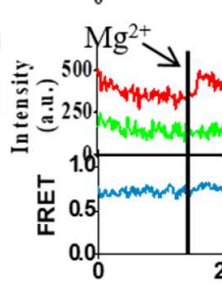

Time (s)

$$
\mathrm{Mg}^{2+}
$$

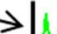

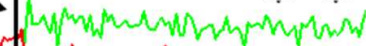

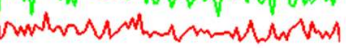

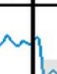
Time (s) $-\mathrm{Cy} 3-\mathrm{Cy} 5$ Mrwillinimb ( P1 namurners 


\section{Selence Submitted Manuscript: Confidential}

\section{DIAAAS}

Figure 3
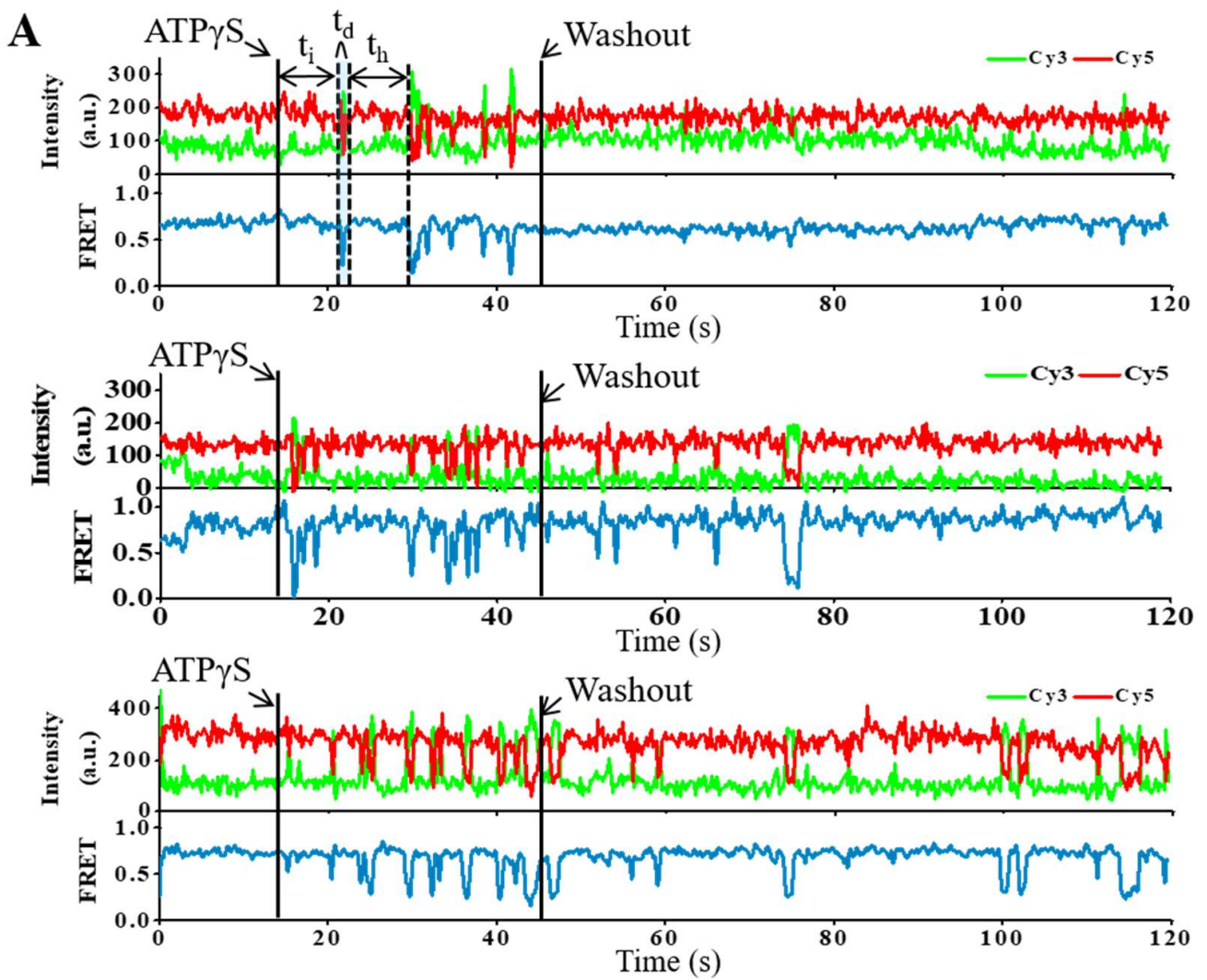

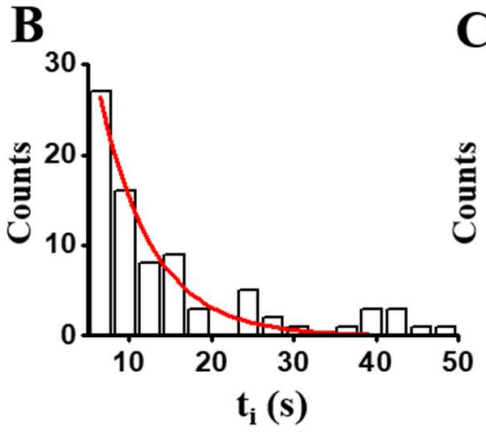

$\mathbf{E}$

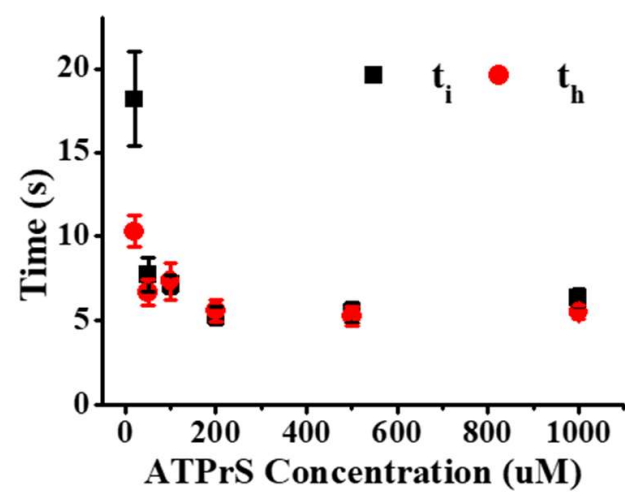

D

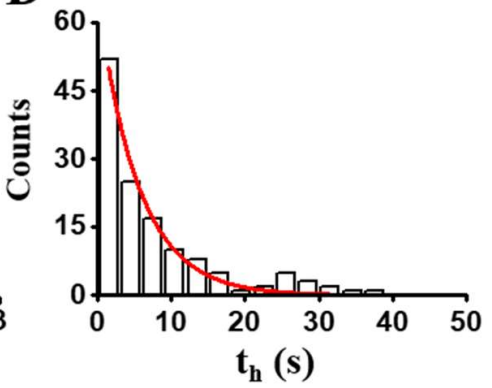

$\mathbf{F}$

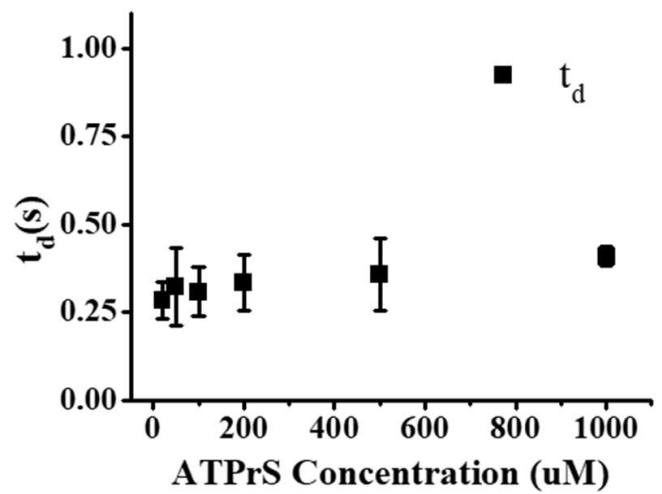


Selence Submitted Manuscript: Confidential

MIAAAS

Figure 4
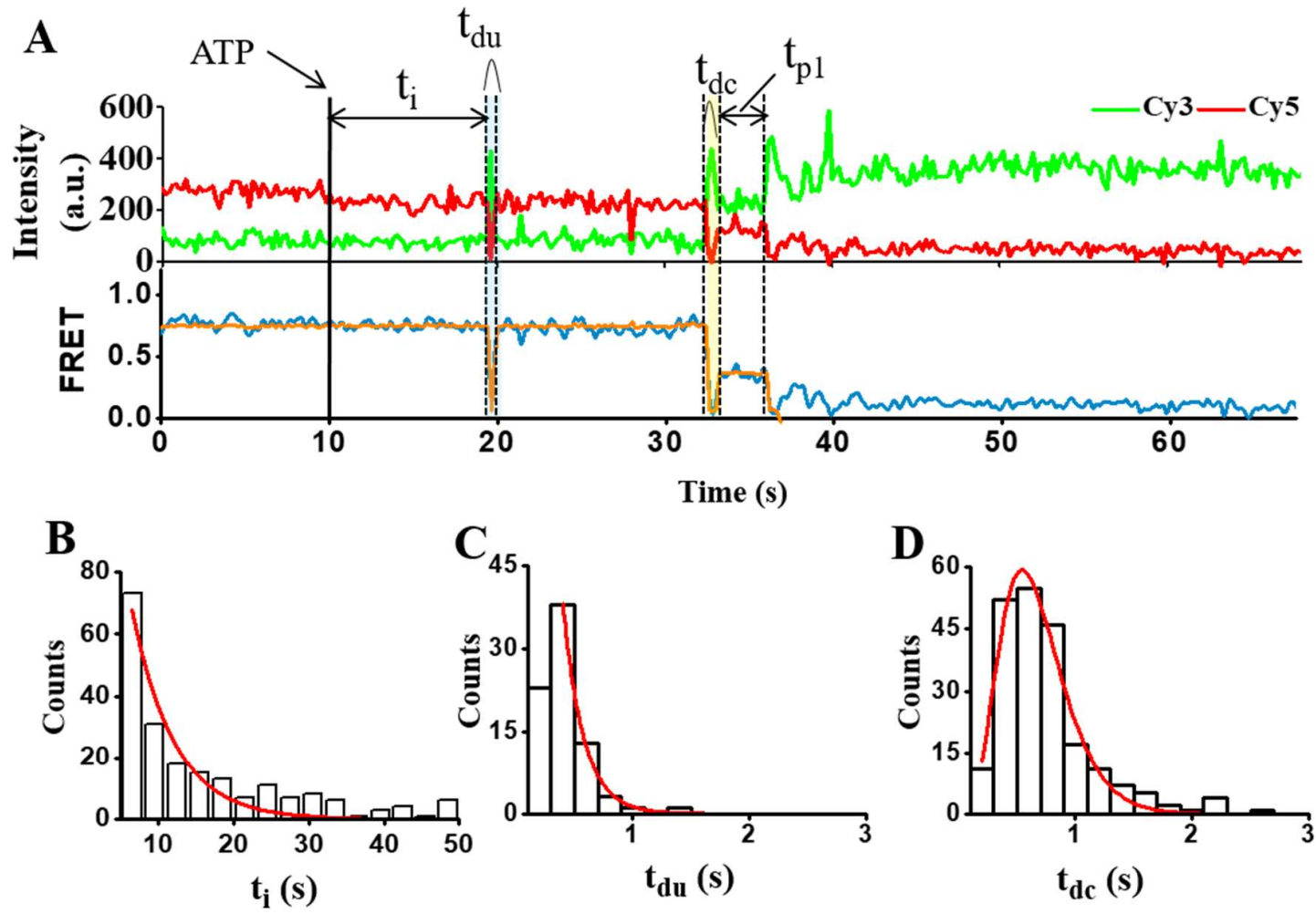

$\mathbf{E}$

$\mathbf{F}$
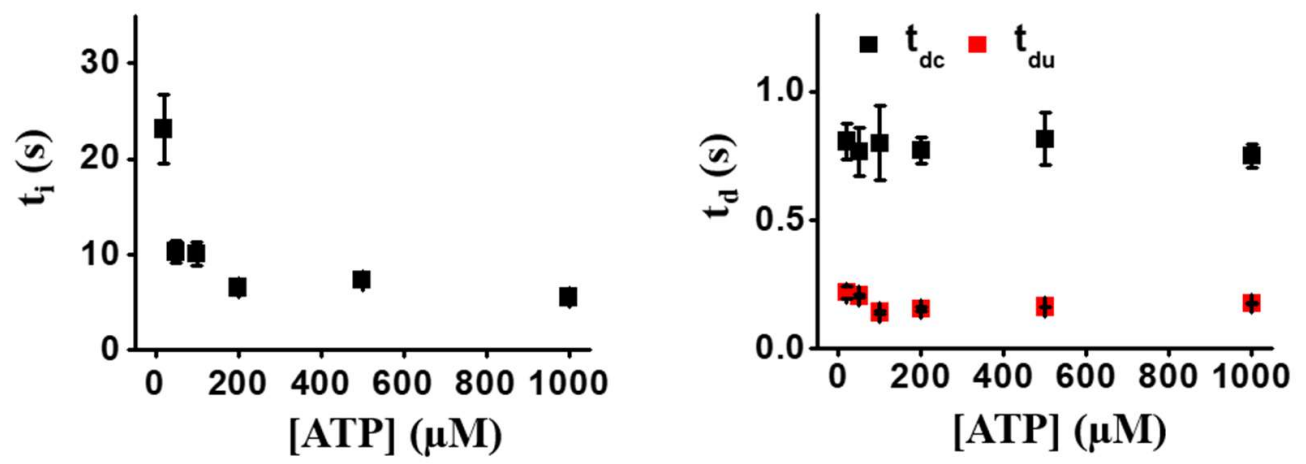

G

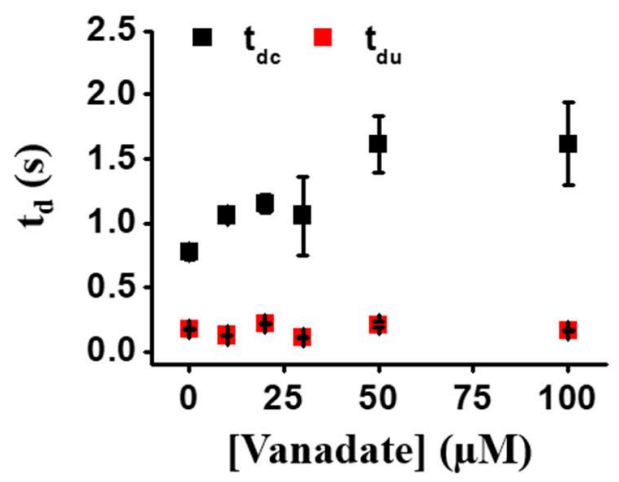

H

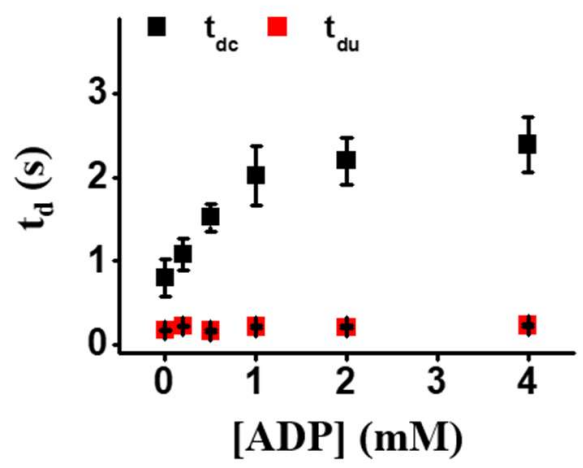




\section{Selence Submitted Manuscript: Confidential}

\section{AlAAAS}

Figure 5
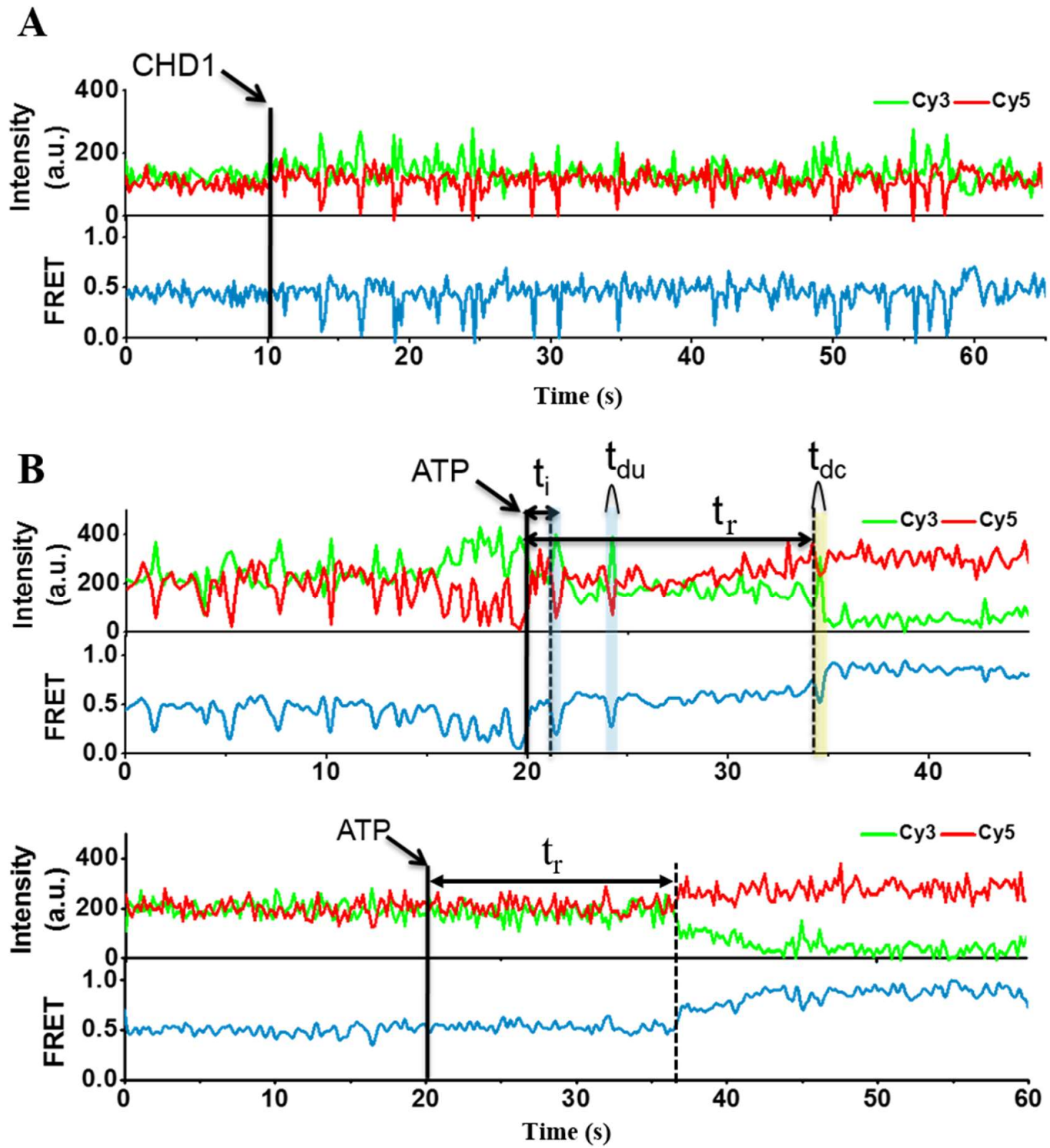

C

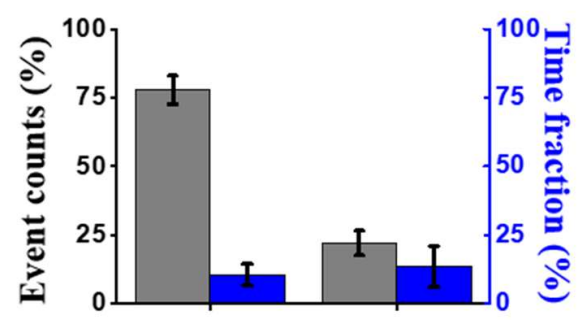

Exit Entry
D

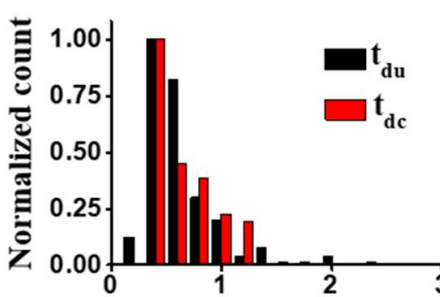

Time (s)
$\mathbf{E}$

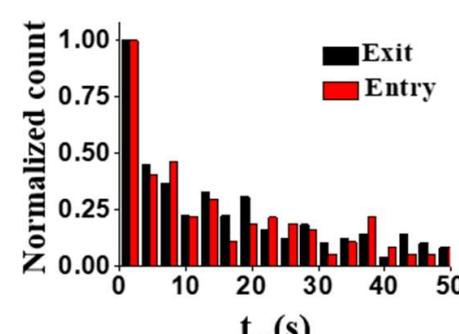


Science

DIAAAS

Figure 6

A

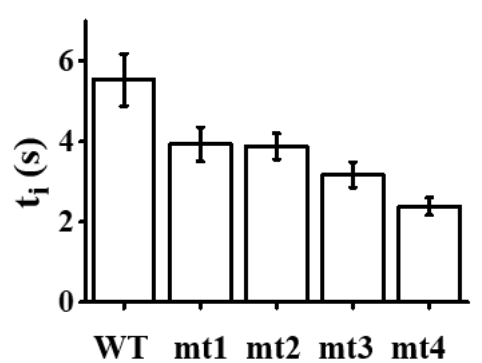

WT mt1 mt2 mt3 mt4
B

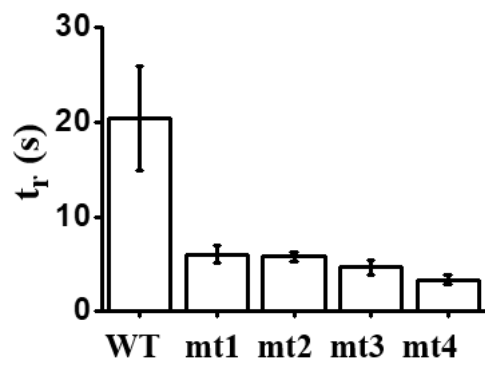

C

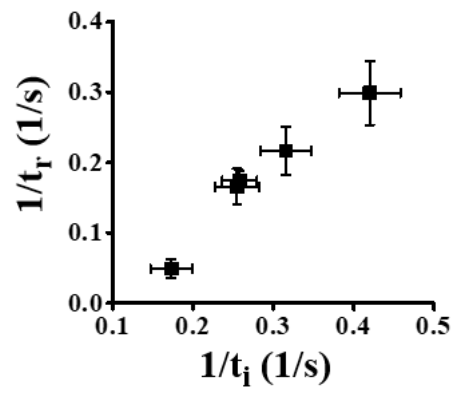

D

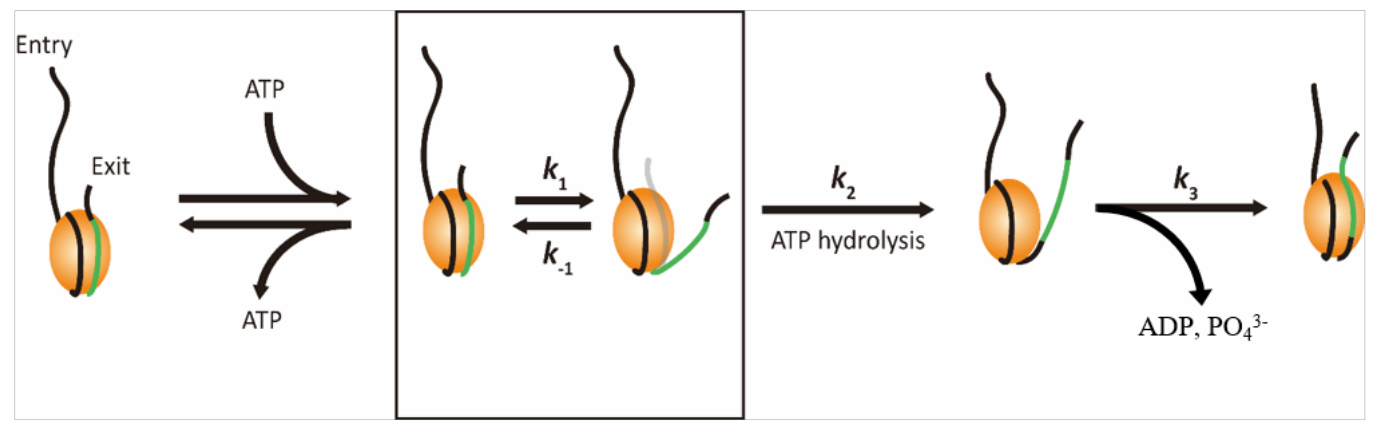

$\mathbf{E}$

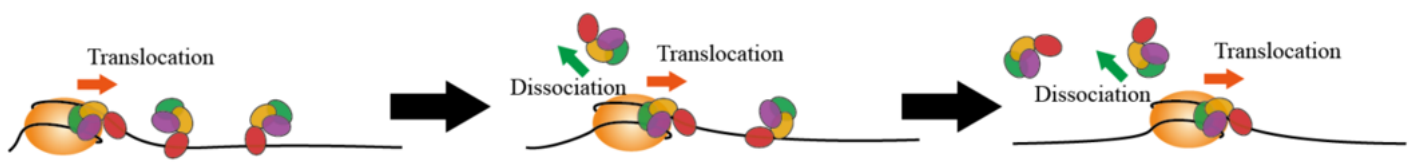




\section{SUPPLEMENTARY INFORMATION}

TITLE: Yeast CHD1 remodels nucleosomes with unique DNA unwrapping and translocation dynamics

Jaewon Kirk ${ }^{1 \dagger}$, Ju Yeon Lee ${ }^{1 \dagger}$, Yejin Lee ${ }^{2 \dagger}$, Soochul Shin ${ }^{1}$, Eunhye Lee ${ }^{2}$, Ji-Joon Song ${ }^{2 *}$, and Sungchul Hohng ${ }^{*}$

${ }^{1}$ Department of Physics and Astronomy, Institute of Applied Physics, Seoul National University, Seoul, Republic of Korea.

${ }^{2}$ Department of Biological Sciences, KI of the BioCentury, Korea Advanced Institute of Science and Technology (KAIST), Daejeon, Republic of Korea.

*Correspondence should be addressed to songj@kaist.ac.kr (J. S.) or shohng@snu.ac.kr (S. H.)

$\dagger$ These authors contributed equally to this work. 


\section{Materials and Methods}

\section{Protein expression, and purification.}

Yeast Chd1 cDNA was cloned into pFastBac vector for expression in Sf9 insect cells. N-terminal His-tagged Chd1 was transduced in Sf9 via baculovirus system. Transduced cells were cultured for $45 \mathrm{~h}$, harvested and resuspended in a buffer containing $300 \mathrm{mM} \mathrm{NaCl}, 5 \%$ Glycerol, $50 \mathrm{mM}$ Tris pH 8.0, and cOmplete ${ }^{\mathrm{TM}}$, Mini, EDTA-free Protease Inhibitor Cocktail (Roche). His-tagged Chd1 was purified by Ni-NTA agarose affinity chromatography (Qiagen) and treated with homemade TEV protease to remove the tag, followed by ion exchange and size exclusion chromatography (GE Healthcare) in a buffer containing 300mM NaCl, 50mM Tris pH 8.0, $1 \mathrm{mM}$ DTT and 20\% Glycerol. The SNAP tagged Chd1p was also expressed in Sf9 insect cells and purified as the wild-type Chd1p. Xenopus laevis histones including H2A T120C were cloned into pET vector and expressed in E.Coli (BL21 DE3 pLyss) as an insoluble form. Each histone in inclusion body was unfolded in a buffer containing $6 \mathrm{M}$ ganidinium $\mathrm{HCl}, 20 \mathrm{mM}$ Na-Ac pH 5.2, and $5 \mathrm{mM}$ DTT and purified by size exclusion and ion exchange chromatography (GE Healthcare). Purified histones were lyophilized and resolved with the same molecular ratio in the buffer used for unfolding process. Resolved histones were mixed with each other and dialyzed in a buffer containing $2 \mathrm{M} \mathrm{NaCl}, 10 \mathrm{mM}$ Tris $\mathrm{pH}$ 7.5, 1 mM EDTA, and $5 \mathrm{mM} \beta$-mercaptoethanol to be assembled into histone octamer.

\section{DNA and nucleosome preparation.}

The standard nucleosomes used in this work were designed to have a 78-bp spacer on the entry side and a 3-bp spacer on the exit side (Figure S1A). DNA fragments containing 601 nucleosome positioning sequence (37) were generated by PCR as described (33). Primers with Cy5 at the 5', end, internal amine modification, or biotin were purchased from IDT. The amine modified primers were labeled with Cy5 NHS ester. PCR products were purified by $5 \%$ native PAGE gel. Nucleosomes were reconstituted with histone octamer containing either Cy3-labeled H2A T120C or Cy3-labeled H4 R45C and Cy5-labeled DNA fragment using salt gradient dialysis.

\section{Single-molecule FRET experiment.}

Quartz slides and coverslips were cleaned with piranha solution (mixture of 3:1 concentrated sulfuric acid:30\% [v/v] hydrogen peroxide solution), coated with aminopropyl silane first, and then with mixture of PEG (m-PEG-5000, Laysan Bio) and biotin-PEG (biotin-PEG-5000, Laysan Bio). A flow cell was assembled by combining a coverslip and a quartz slide with double-sided adhesive tape (3M). For convenient buffer exchange, polyethylene tubes (PE50; Becton 
Dickinson) were connected to the flow cell. Nucleosome substrates were immobilized on a PEGylated surface using biotin-streptavidin conjugation and then incubated with Chd1 $\mathrm{p}(180 \mathrm{nM})$ for 2-3 minutes. Single-molecule FRET experiments were performed in an imaging buffer (10 $\mathrm{mM}$ Tris- $\mathrm{HCl} \mathrm{pH}$ 8.0, $100 \mathrm{mM} \mathrm{KCl}, 3 \mathrm{mM} \mathrm{MgCl} 2,5 \mathrm{mM} \mathrm{NaCl}$ ) containing a gloxy oxygen scavenging system (a mixture of glucose oxidase $(1 \mathrm{mg} / \mathrm{ml}$, Sigma), catalase $(0.04 \mathrm{mg} / \mathrm{ml}$, Sigma), glucose (0.4\% (w/v), Sigma), and Trolox ( $2 \mathrm{mM}$, Sigma)). During imaging, various concentration of ATP or ATP analog was injected with imaging buffer to initiate the reactions by Chd1p. For $\mathrm{Mg}^{2+}$ flow experiment, surface immobilized nucleosomes were incubated with 180 nM Chd1p and $100 \mu \mathrm{M}$ ATP in imaging buffer (with $10 \mathrm{mM}$ EDTA instead of $\mathrm{MgCl}_{2}$ ), and then an imaging buffer containing $3 \mathrm{mM} \mathrm{MgCl} 2$ was injected during imaging. Single-molecule fluorescence images were acquired at a frame rate of $10 \mathrm{~Hz}$ using a home-built prism-type total internal reflection fluorescence microscope equipped with an electron-multiplying charge coupled device camera (Ixon DV897; Andor Technology). Cy3 and Cy5 were alternately excited with $532 \mathrm{~nm}$ and $633 \mathrm{~nm}$ lasers using the ALEX (Alternative Laser EXcitation) technique (35).

Experimental temperature was maintained at $30^{\circ} \mathrm{C}$ using a temperature control system (Live Cell Instruments). Data acquisition and FRET trace extraction were done using homemade programs written in LabView (National Instruments) and IDL (ITT), respectively. The intensity traces of Cy3 and Cy5 were analyzed using a custom made Matlab (MathWorks) script. The FRET efficiencies were calculated from the donor (ID) and acceptor (IA) fluorescence intensities as $\mathrm{EFRET}=\mathrm{IA} /(\mathrm{IA}+\mathrm{ID})$. 
A

Exit

GCCCT GGAGA ATCCC GGTCT GCAGG CCGCT CAATT GGTCG TAGAC AGCTC TAGCA CCGCT TAAAC GCACG TACGC GCTGT CCCCC GCGTT TTAAC CGCCA AGGGG ATTAC TCCCT AGTCT CCAGG CACGT GTCAG ATATA TACAT CCTGT GCATG TATTG AACAG CGACC TTGCC GGTGC CAGTC GGATA GTGTT CCGAG CTCCC ACTCT AGAGG ATCCC CGGGT ACC

Entry

GCCCT GGAGA ATCCC GGTCT GCAGG CCGCT CAATT GGTCG TAGAC AGCTC TAGCA CCGCT TAAAC GCACG TACGC GCTGT CCCCC GCGTT TTAAC CGCCA AGGGG ATTAC TCCCT AGTCT CCAGG CACGT GTCAG ATATA TACAT CCTGT GCATG TATTG AACAG CGACC TTGCC GGTGC CAGTC GGATA GTGTT CCGAG CTCCC ACTCT AGAGG ATCCC CGGGT ACC

B

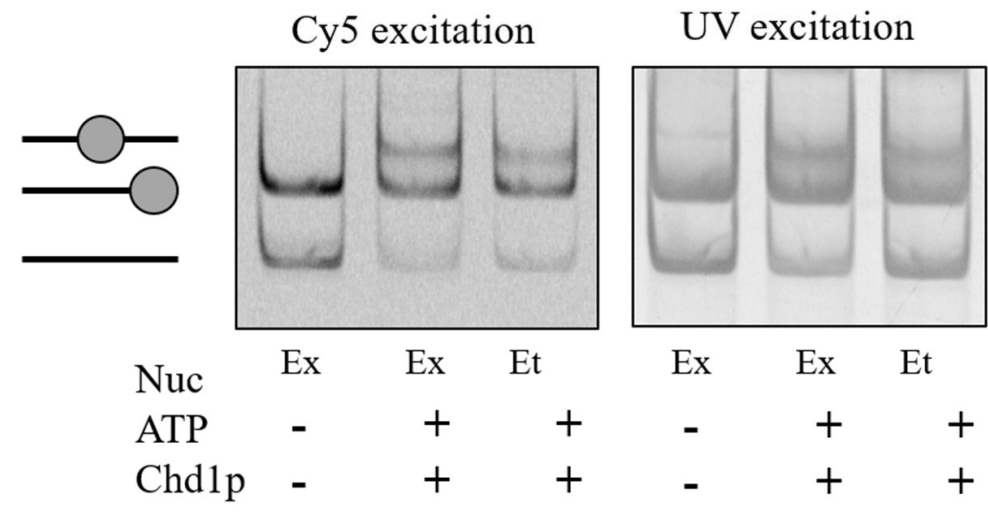

Figure S1. DNA sequences and remodeling activity of Chd1p on labelled

nucleosomes. (A) DNA sequences used in the study. The DNA fragments consist of 601 nucleosome positioning sequence (black) with the 3 bp linker on the exit side and the 78 bp on the entry side (blue). The Cy5 labeling position is marked in red. (B) Remodeling activity of Chd1p on fluorophore-labeled nucleosomes. Bulk remodeling assays were carried out in $10 \mathrm{mM}$ Tris- $\mathrm{HCl} \mathrm{pH} 8.0$ with $100 \mathrm{mM} \mathrm{KCl}, 3 \mathrm{mM} \mathrm{MgCl}_{2}, 5 \mathrm{mM} \mathrm{NaCl}, 2.5$ $\mathrm{mM}$ ATP, $700 \mathrm{nM}$ of Chd1p, and $280 \mathrm{nM}$ nucleosomes at $30^{\circ} \mathrm{C}$ for $20 \mathrm{~min}$. The products were examined using a 5\% native PAGE gel. (Nuc, Nucleosome; Ex, exit-side nucleosome; Et, entry-side nucleosome) 

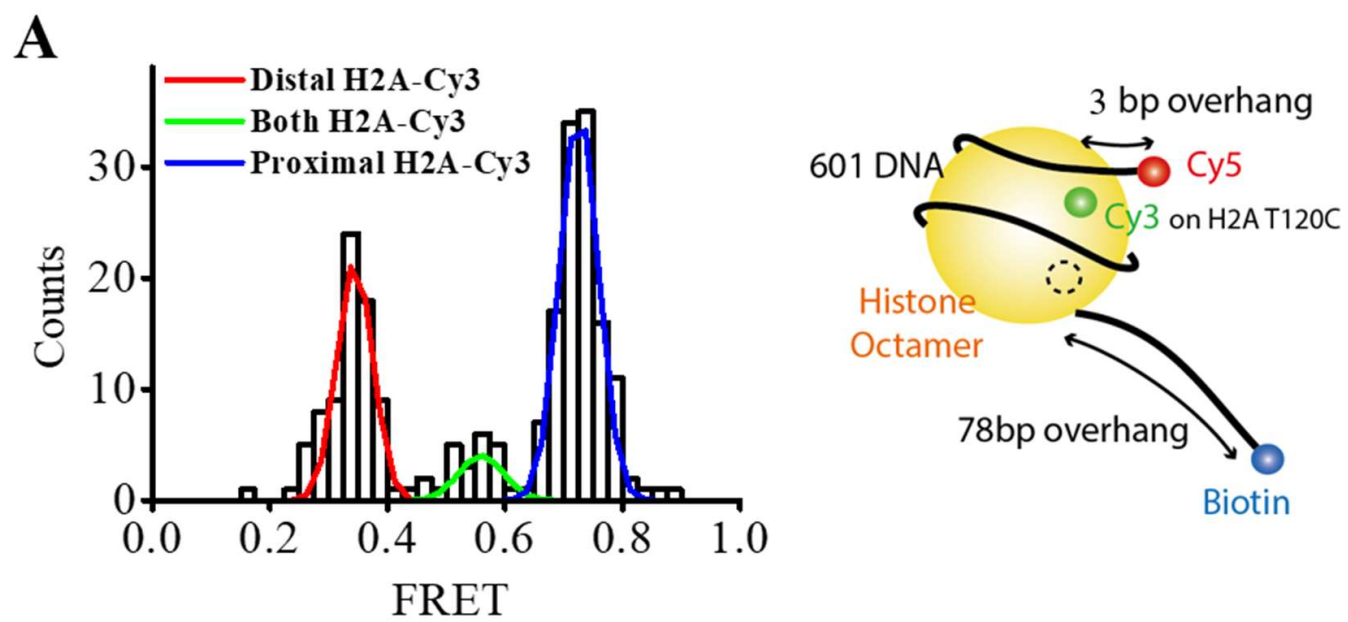

Figure S2. FRET histogram of the exit-labeled nucleosome. (A) FRET histogram and labeling scheme of the exit-side labelled nucleosomes. The histogram shows three FRET peaks. The low and high FRET corresponds to species labeled at the distal and proximal H2A, respectively. The mid FRET corresponds to doubly-labeled species. We selected only high FRET species for data analysis. 

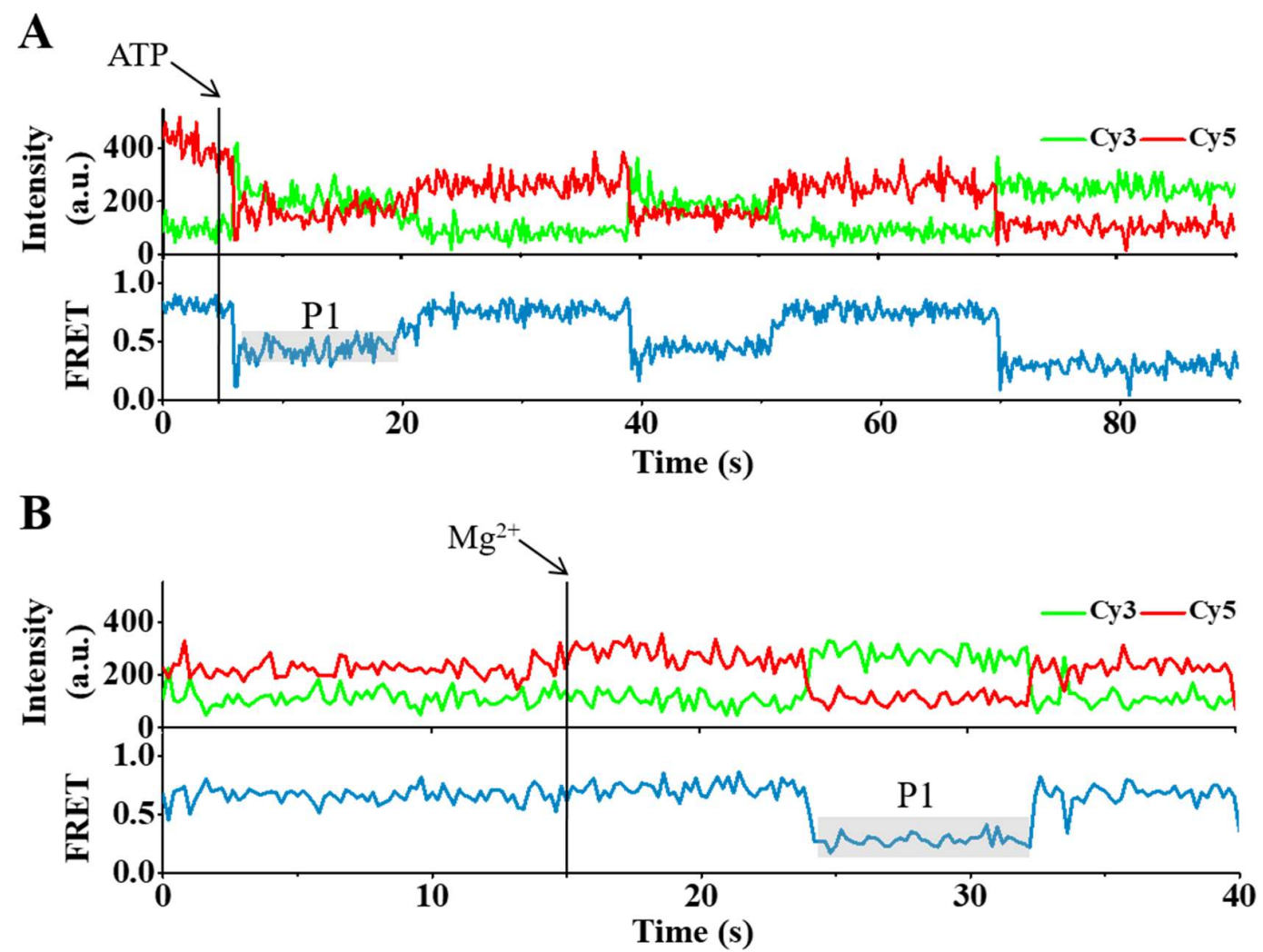

Figure S3. Bidirectional nucleosome remodeling by Chd1p. (A) A representative fluorescence and FRET time traces showing back-and-forth translocation of nucleosome on the exit side by CHD1. The reaction was started by injecting ATP. (B) A representative fluorescence and FRET time trace showing back-and-forth translocation on the exit side. Chd1p-nucleosome complex was preincubated with ATP and EDTA, and the remodeling reaction was initiated by injecting $\mathrm{Mg}^{2+}$. 
A
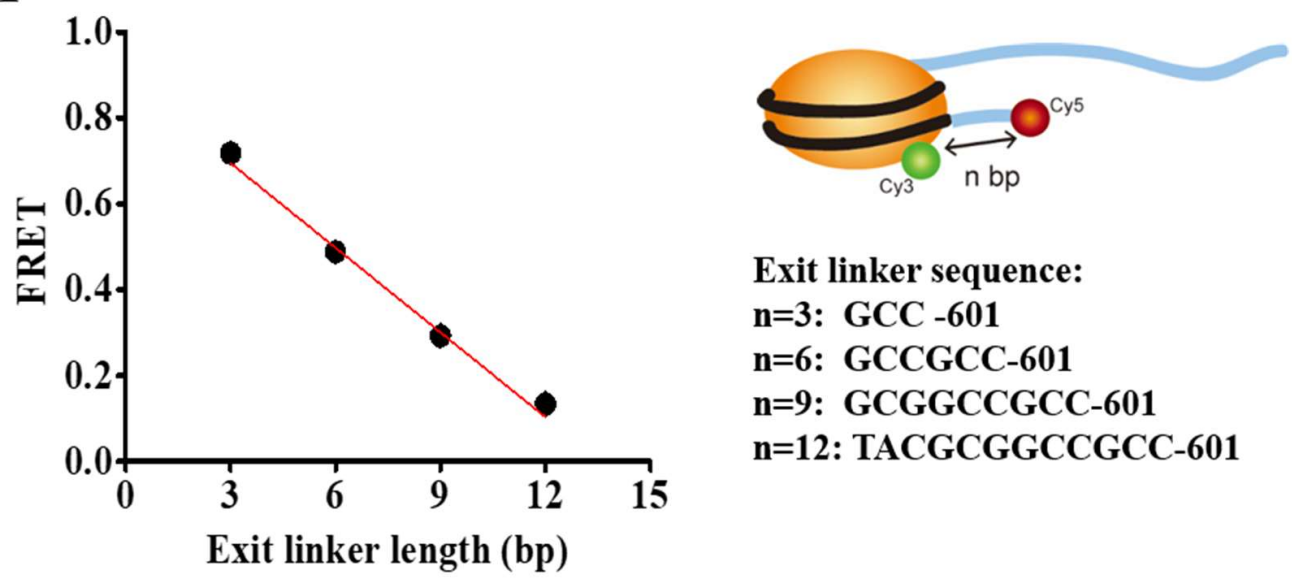

Exit linker sequence:

n=3: GCC -601

n=6: GCCGCC-601

n=9: GCGGCCGCC-601

n=12: TACGCGGCCGCC-601

Figure S4. Linear relation between FRET efficiency and the exit linker length. (A)

Calibration data for step size determination. FRET was obtained from nucleosomes with varying linker DNA lengths $(\mathrm{n}=3,6,9,12 \mathrm{bps})$ on the exit side. The linear-fit of the data had a slope of $-0.066 \pm 0.0004$ per base pair. 
A

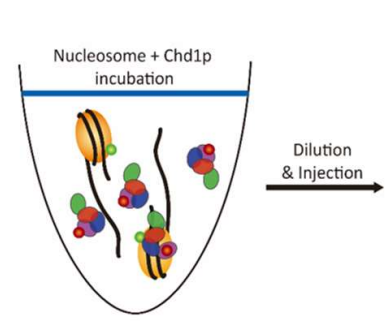

B

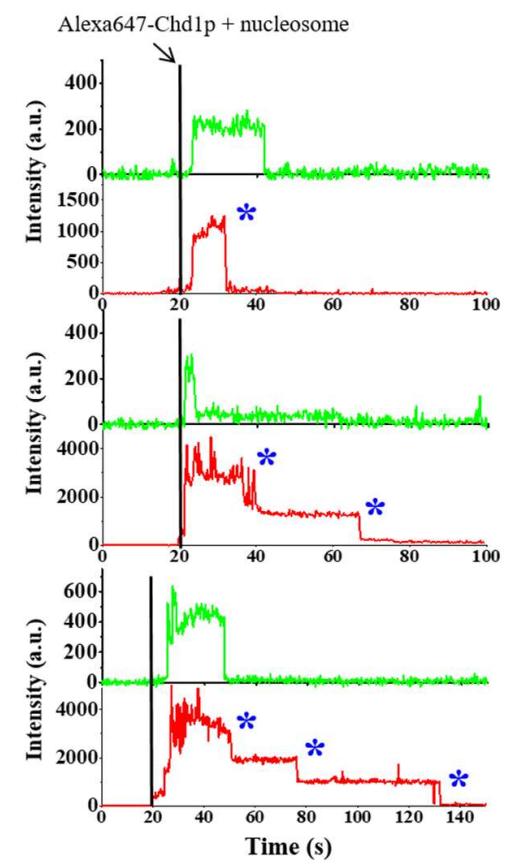

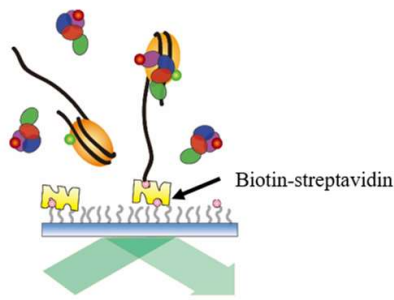

C

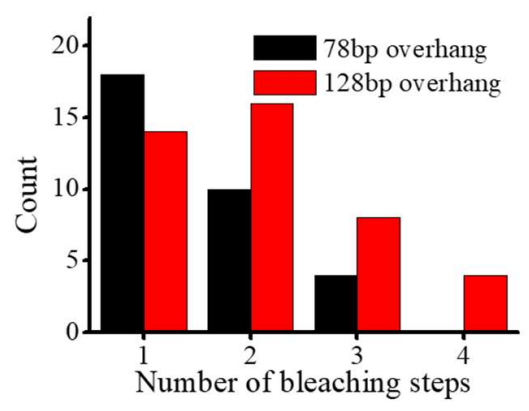

Figure S5. The number of Chd1p on a single nucleosome. (A) Experimental scheme for Chd1p counting. Mixture of 15nM Cy3-labeled Nucleosome and 200nM Alexa647-labeled Chd1 $\mathrm{p}$ in T50 buffer ( $50 \mathrm{mM} \mathrm{NaCl}, 10 \mathrm{mM}$ Tris- $\mathrm{HCl}(\mathrm{pH} 8.0)$ ) was incubated for 3 minutes, and 100x diluted with the imaging buffer. The diluted sample was injected into the imaging chamber while single-molecule images were being taken. Binding of a nucleosome/Chd1p complex on surfaceimmobilized streptavidin was monitored using single-molecule fluorescence microscope. (B) Representative fluorescence intensity time traces of $\mathrm{Cy} 3$ (green) and Alexa647 (red) showing the binding events (simultaneous appearance of $\mathrm{Cy} 3$ and Alexa647). Photobleaching steps of Alexa647 are indicated by blue asterisks. (C) Distribution of Alexa647 photobleaching step number observed in (B), using a DNA substrate with a 78bp DNA overhang, the one described in figure S1A (black), and a DNA substrate with a 128bp DNA overhang (red). Based on the distribution and the labeling efficiency of Alexa647 (80\%), the fractions of a nucleosome with one, two, and three Chd lps were estimated as $47.8 \%, 30.4 \%$, and $21.7 \%$, respectively for the case of 78bp DNA overhang sample. 


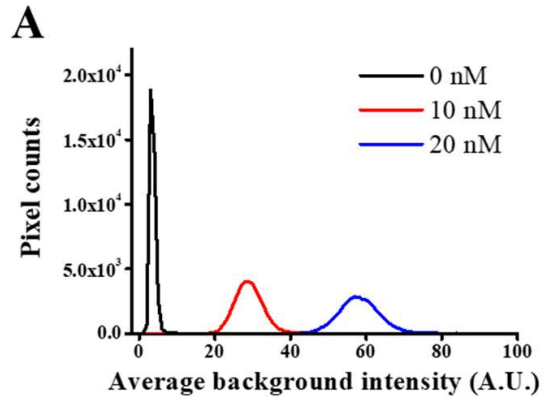

B

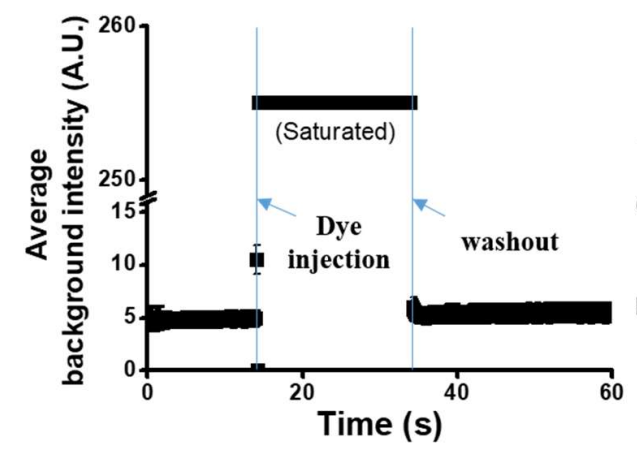

D

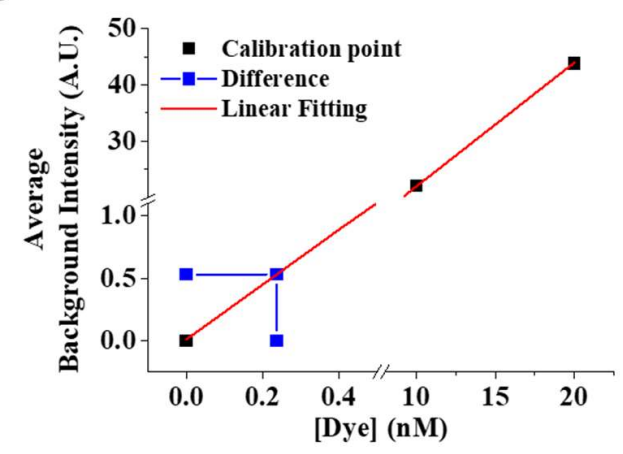

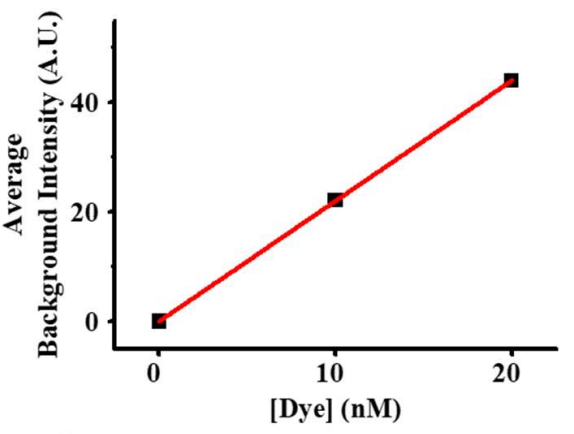

C

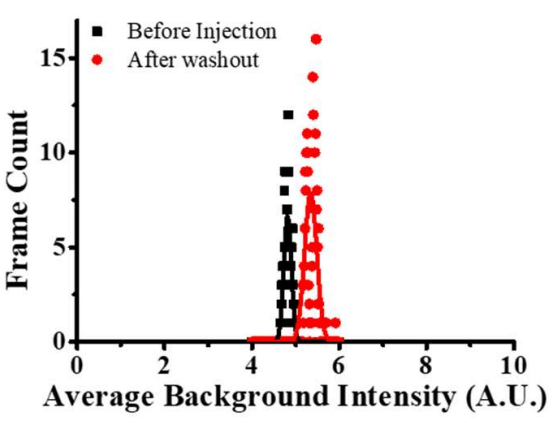

Figure S6. Characterization of the buffer exchange efficiency of the flow system I. (A) Histograms of EM-CCD pixel count in the presence of $0 \mathrm{nM}, 10 \mathrm{nM}, 20 \mathrm{nM}$ free Cy5 (left). The average background intensity and Cy5 concentration exhibited a nice linear relation (right). (B) Average background intensity before the injection and after the removal of Cy5. Cy5 (500nM) was injected into the chamber at $15 \mathrm{~s}$, and then the chamber was washed out with buffer containing no Cy5 (volume: $300 \mu$, flow rate: 100 $\mu \mathrm{l} / \mathrm{s}$ ) at 35s. (C) Background intensity distribution before the injection (black) and after washout of Cy5 (red). (D) Buffer exchange efficiency. The average background intensity after the washout was 0.53 (blue), corresponding to Cy5 concentration of $0.24 \mathrm{nM}$. Therefore, the buffer exchange system is estimated to remove $99.95 \%$ of solutes. 
A

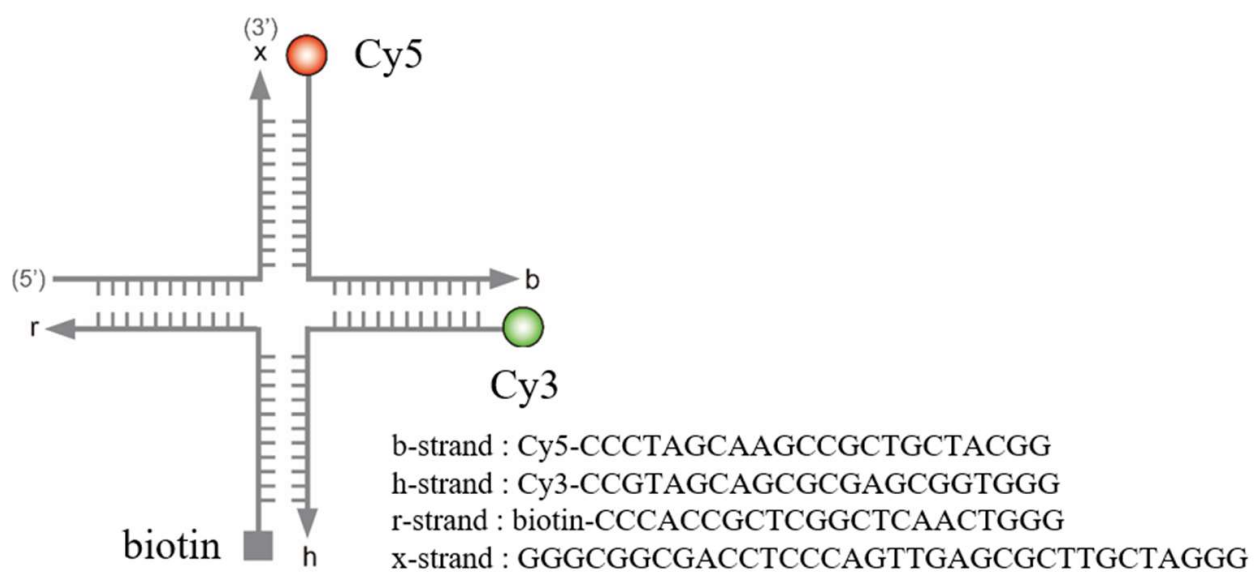

B

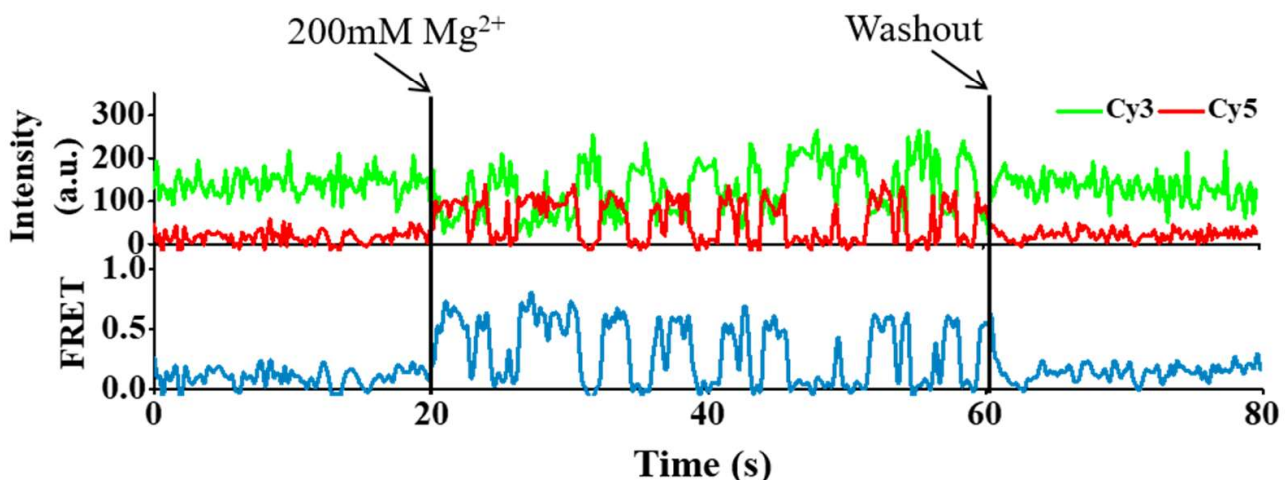

Figure S7. Characterization of the buffer exchange efficiency of the flow system II.

(A) DNA sequences of the Holliday Junction and fluorophore labeling scheme. (B) Representative fluorescence intensity times traces during $200 \mathrm{mM} \mathrm{Mg}^{2+}$ injection and washout with $300 \mu 1$ of $\mathrm{Mg}^{2+}$-free imaging buffer. The absence of FRET dynamics after the washout indicates that $\mathrm{Mg}^{2+}$ remaining after the washout is negligible. 
A

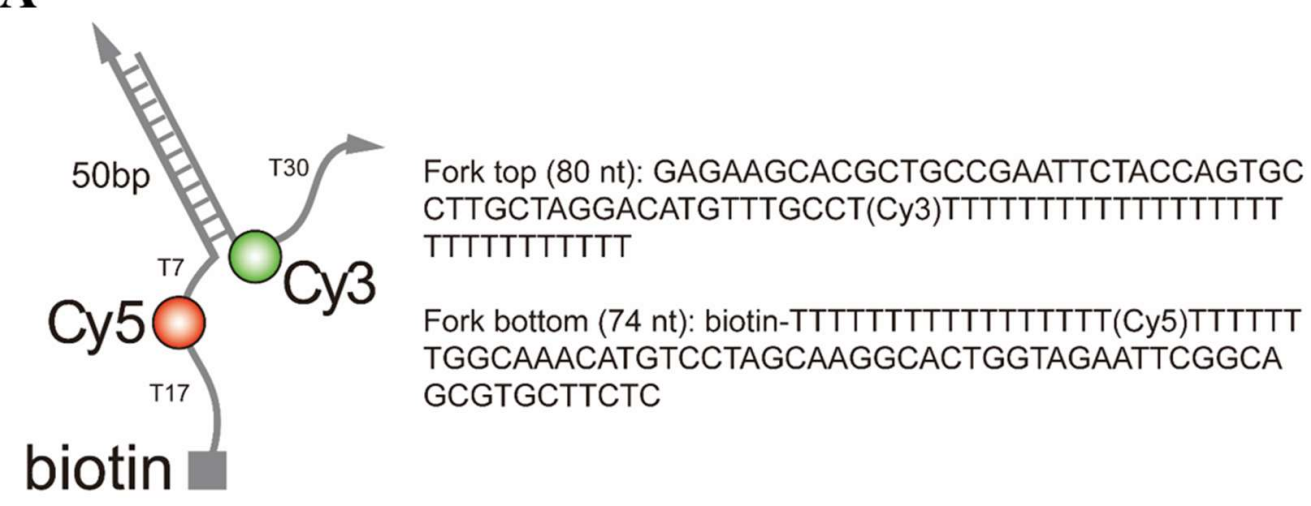

B
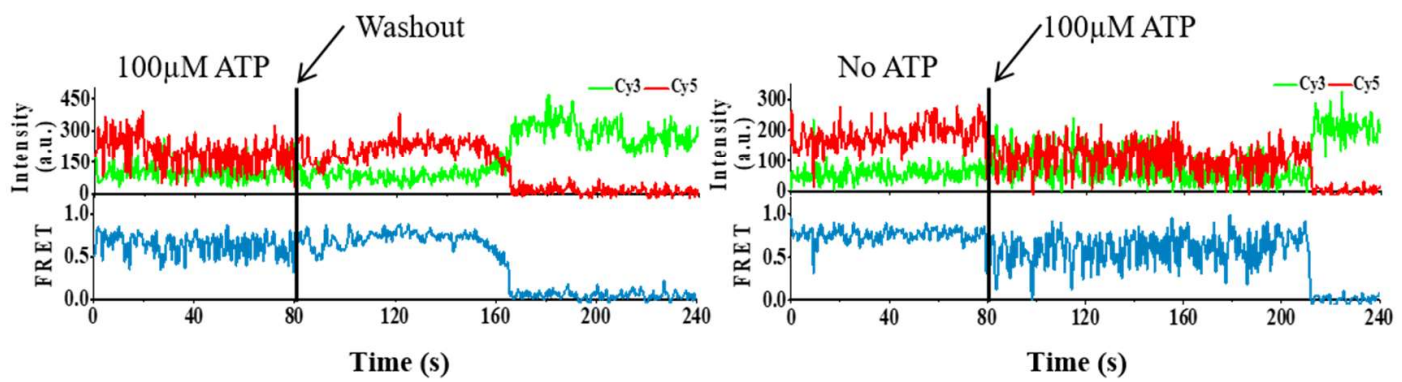

C
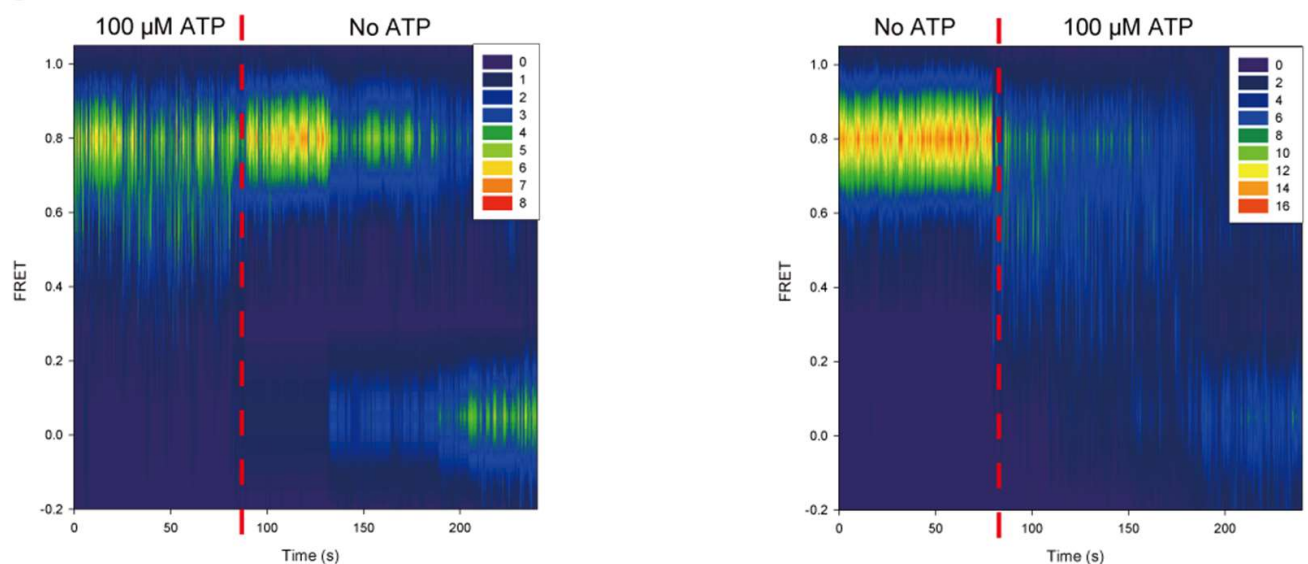

Figure S8. Characterization of the buffer exchange efficiency of the flow system III.

(A) DNA sequences of forked DNA and fluorophore labeling scheme. (B) Representative fluorescence intensity times traces showing repetitive unwinding by HIM-6 (C. elegans BLM helicase ortholog) only in the presence of ATP $(100 \mu \mathrm{M})$. (C) Contour plots of FRET efficiency of the experiments described in (B). Dashed red lines indicate buffer exchange points. 


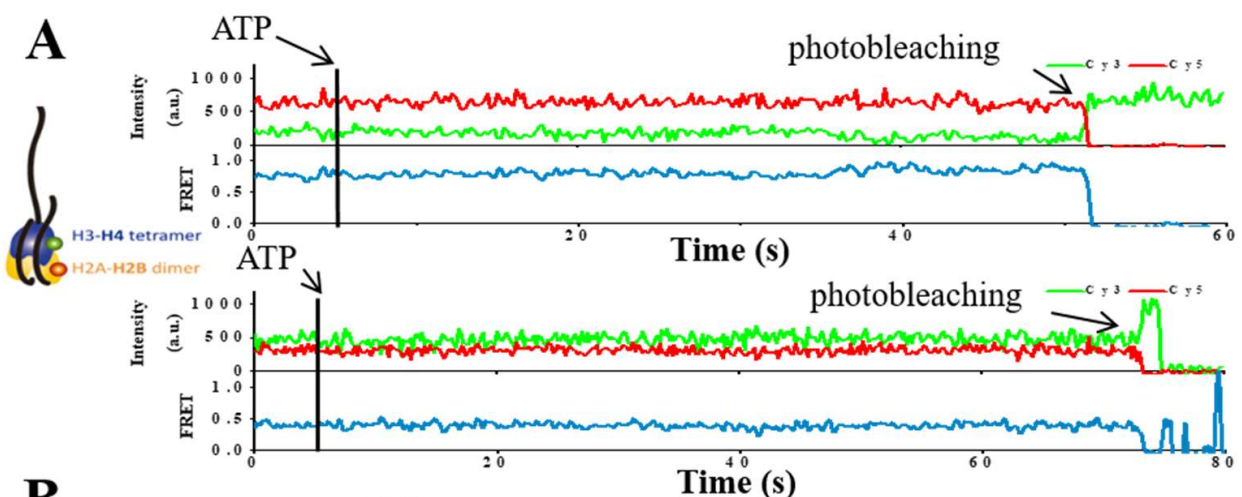

B

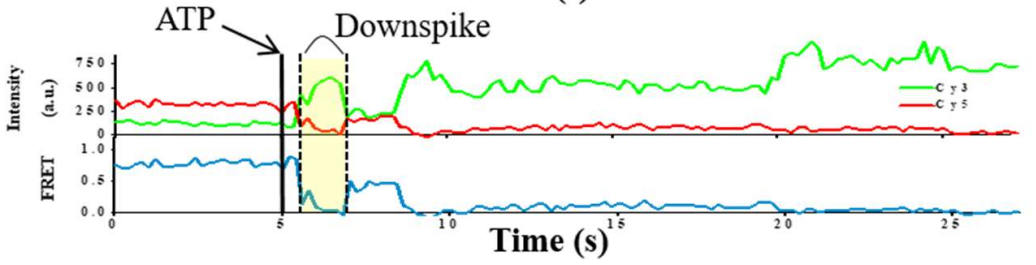

C

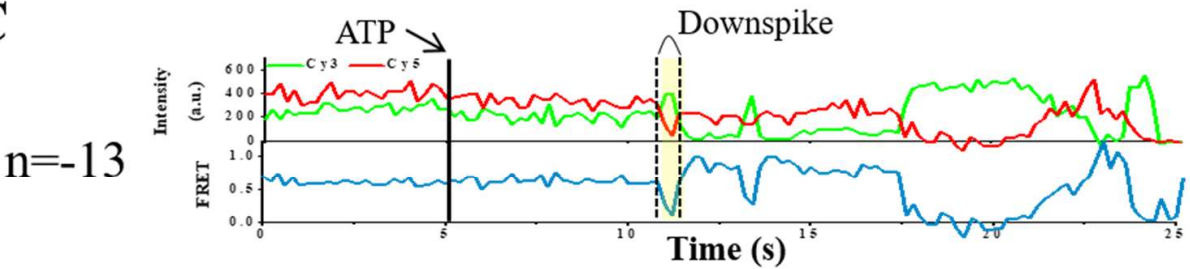

D

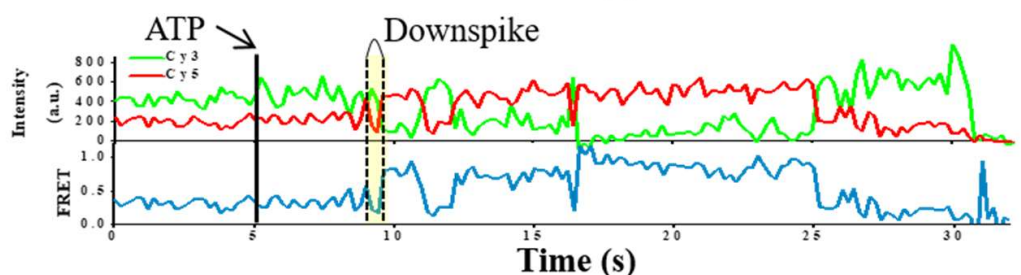

$\mathbf{E}$

Time (s)

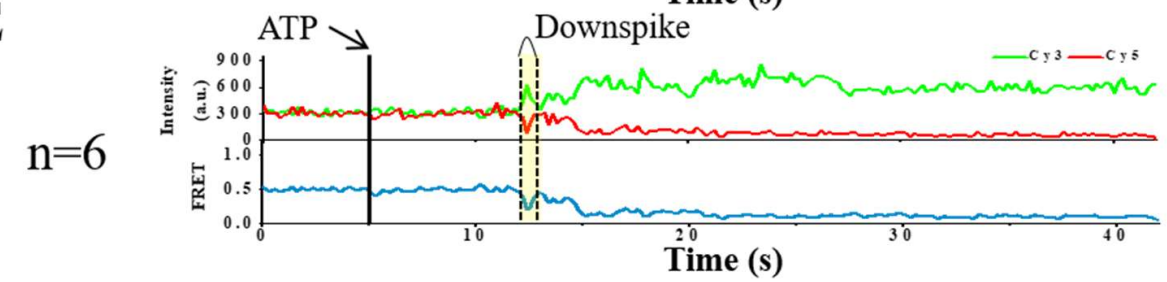

$\mathbf{F}$

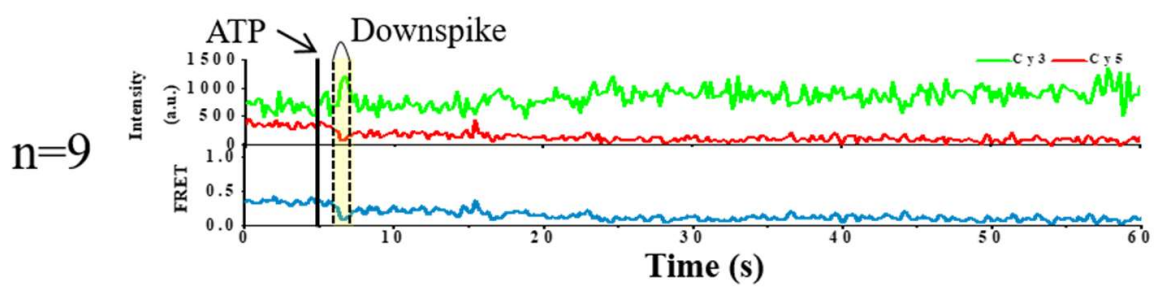

Figure S9. FRET down spikes on the exit side under different labeling schemes. (A)

No dynamics was observed with Cy3 labeled on H4 E63C and Cy5 on H2A T120C. On the other hand, down spikes were observed with (B) Cy3 labeled on H4 R45C and Cy5 on DNA end ( $\mathrm{n}=3$ ), or (C-F) Cy3 labeled on H2A T120C, and Cy5 labeled on several different positions near the exit-side DNA. 


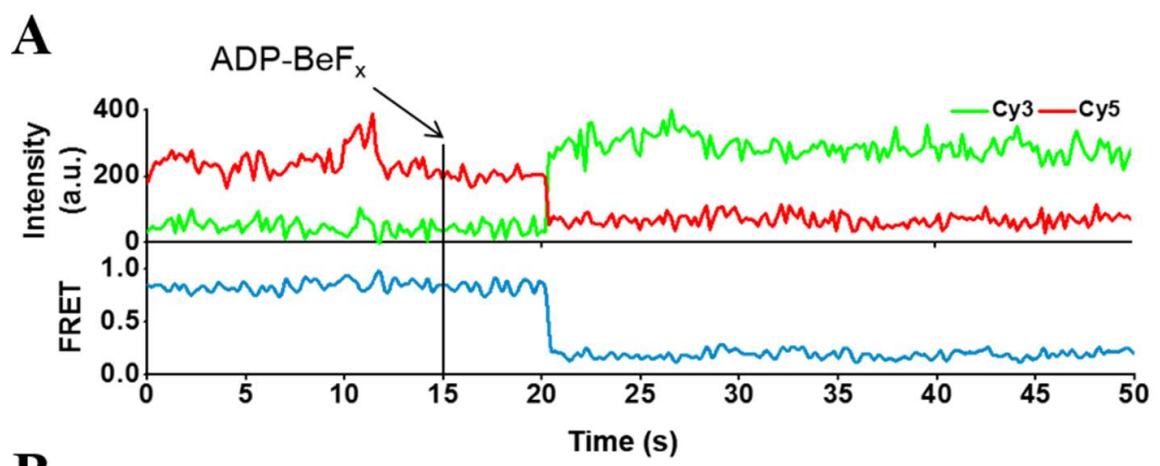

B

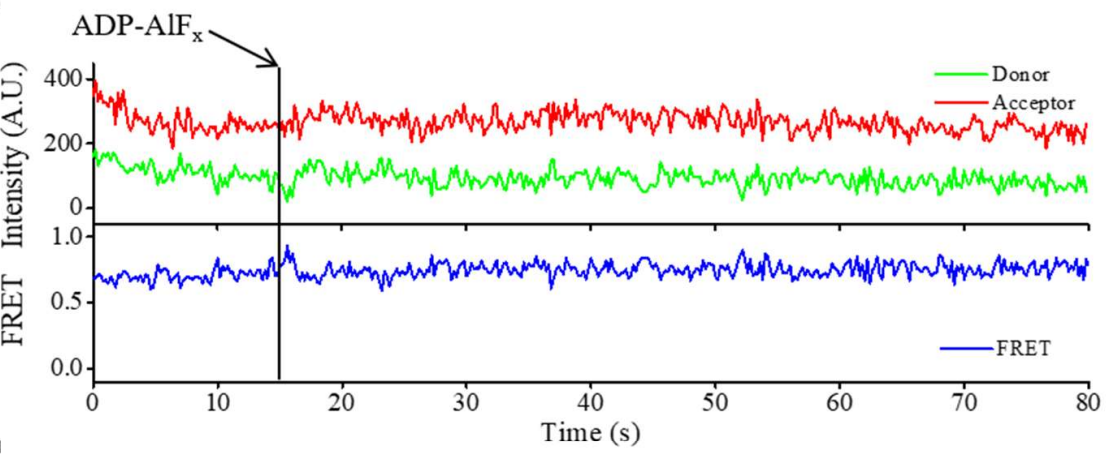

C

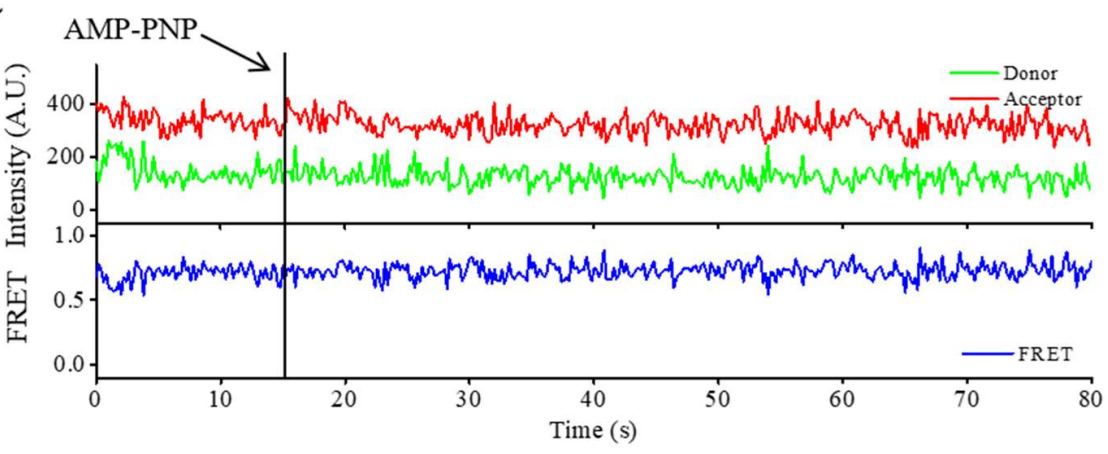

Figure S10. FRET down spikes on the exit side in the presence of various ATP analogs. Stable FRET down spikes were observed in the presence of (A) 1mM ADP$\mathrm{BeF}_{\mathrm{x}}\left(1 \mathrm{mM}\right.$ ADP, $\left.5 \mathrm{mM} \mathrm{BeCl}_{2}, 25 \mathrm{mM} \mathrm{NaF}\right)$, but no down spike was observed in the presence of (B) $1 \mathrm{mM}$ ADP-AlF $\mathrm{x}_{\mathrm{x}}\left(1 \mathrm{mM}\right.$ ADP, $\left.5 \mathrm{mM} \mathrm{AlCl}_{3}, 25 \mathrm{mM} \mathrm{NaF}\right)$, or (C) $1 \mathrm{mM}$ AMP-PNP. 

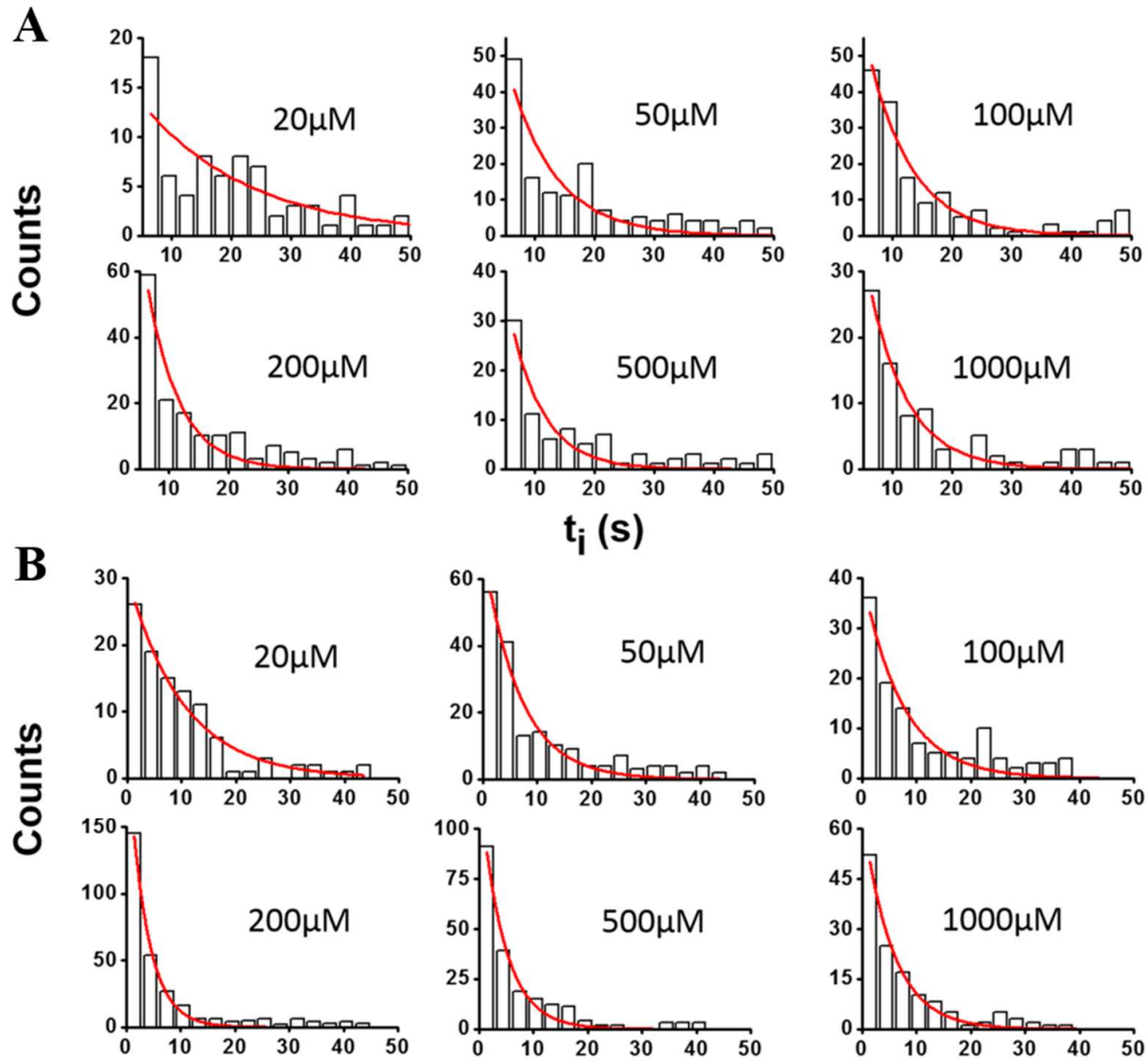

$\mathbf{t}_{\mathbf{i}}(\mathbf{s})$
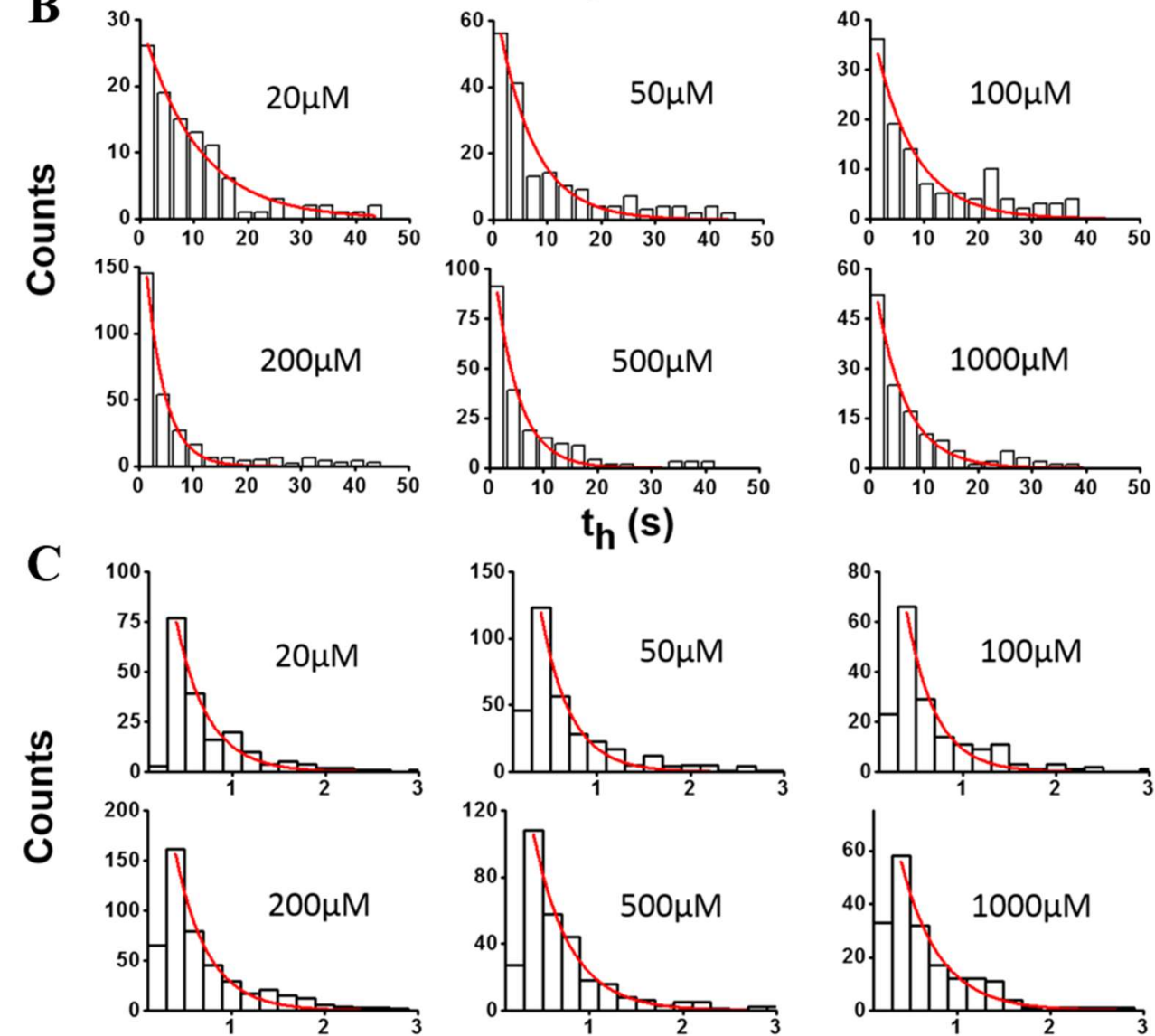

$t_{h}(s)$
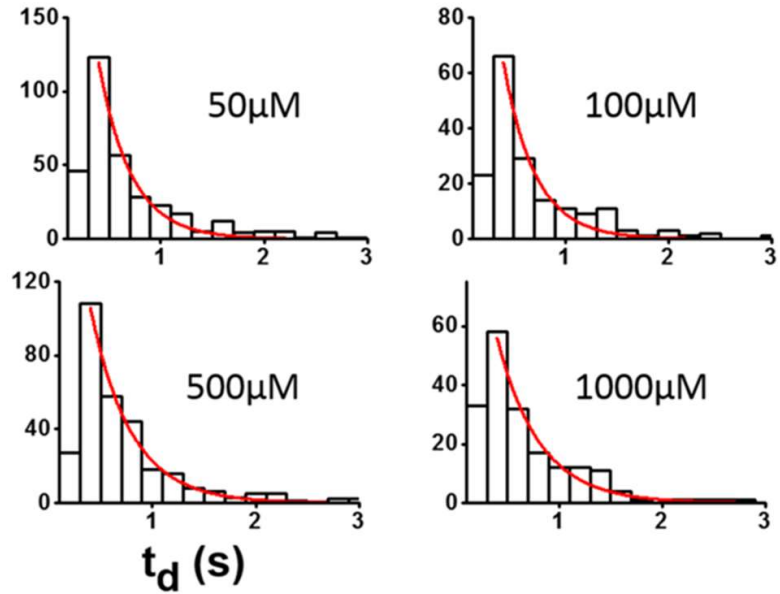

Figure S11. ATP $\gamma \mathrm{S}$ titration of kinetic parameters of the exit side unwrapping. ATP $\gamma \mathrm{S}$ titration of (A) $t_{i}$, (B) $t_{h}$, and (C) $t_{d}$ of the exit side nucleosome, and their fit to single-exponential functions (red lines). 
A
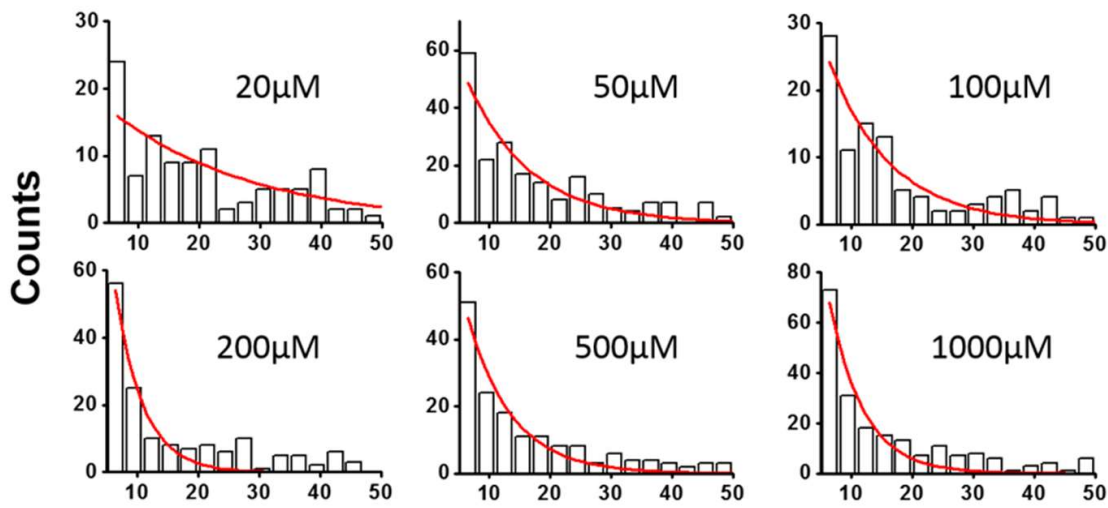

B

$t_{i}(s)$
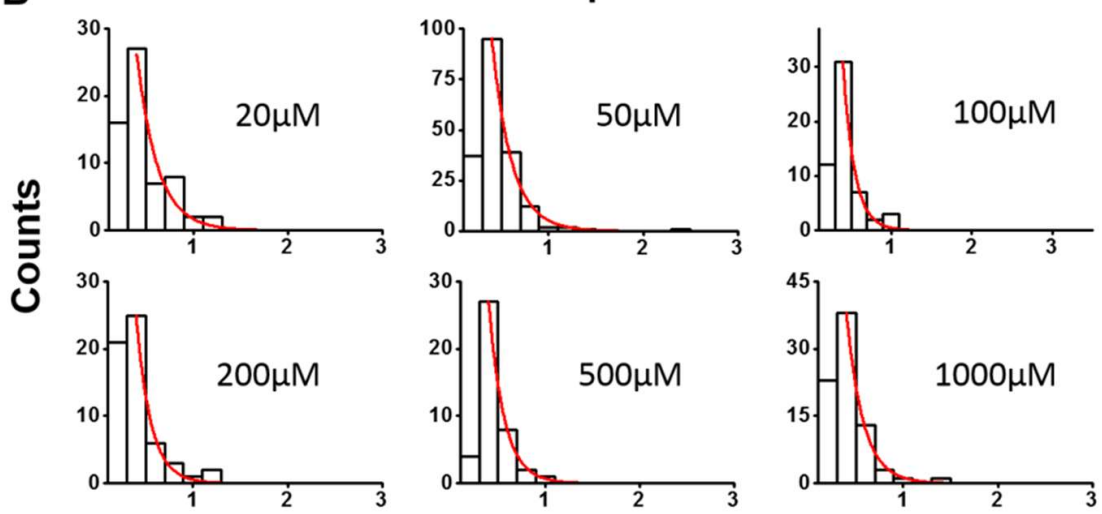

C
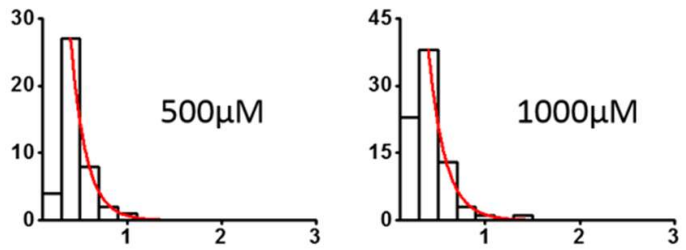

$t_{d u}(s)$
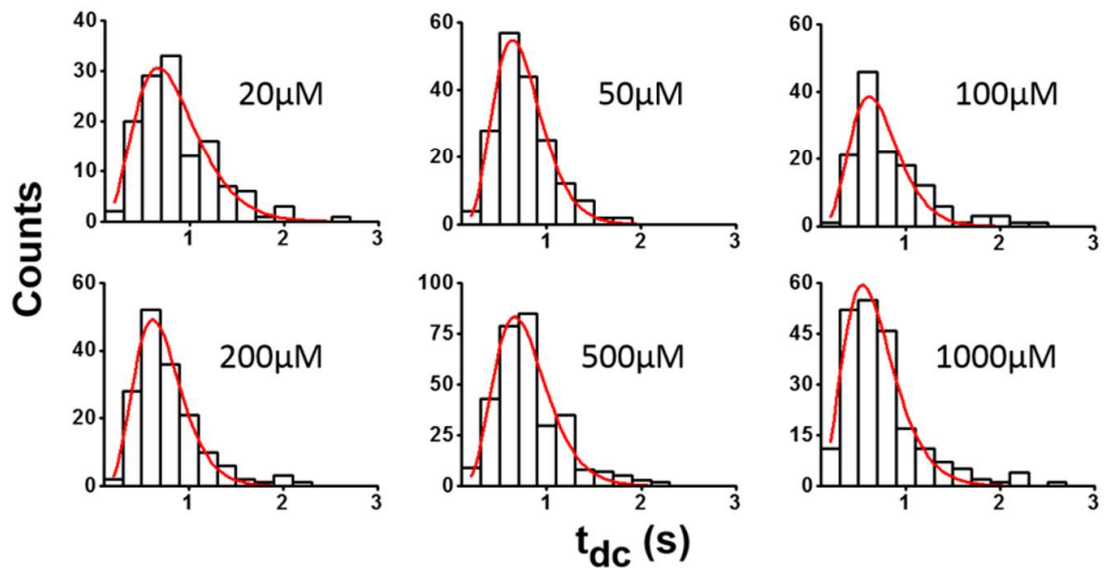

Figure S12. ATP titration of kinetic parameters of the exit side remodeling. ATP titration of $(A) t_{i},(B) t_{d u}$, and (C) $t_{d c}$ of the exit side nucleosome. $t_{i}$ and $t_{d u}$ were fitted to single-exponential functions (red lines). $t_{d c}$ was fitted to gamma functions. 
A ATP + Vanadate

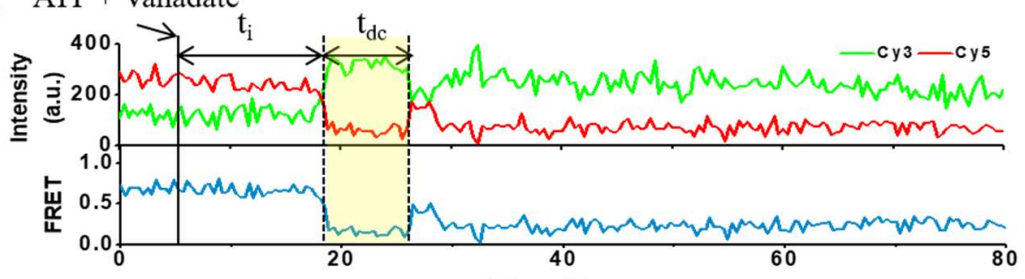

B

Time (s)

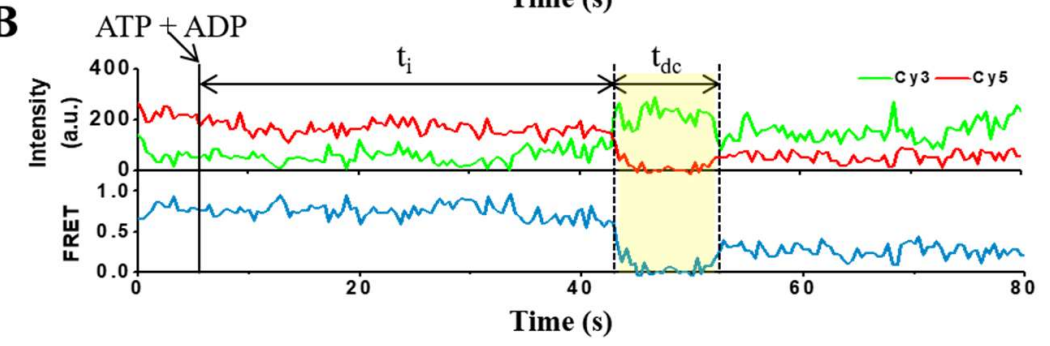

C
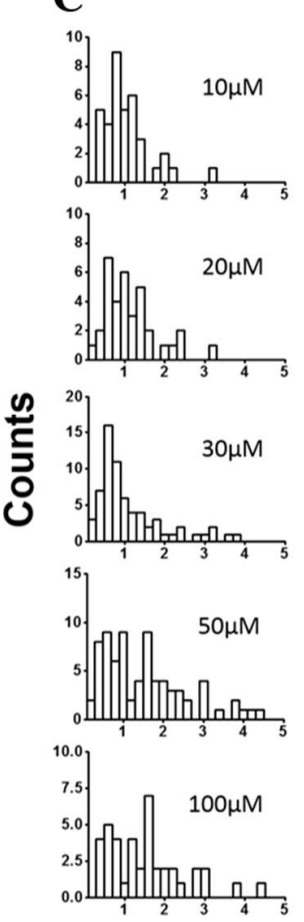

$t_{d c}(s)$
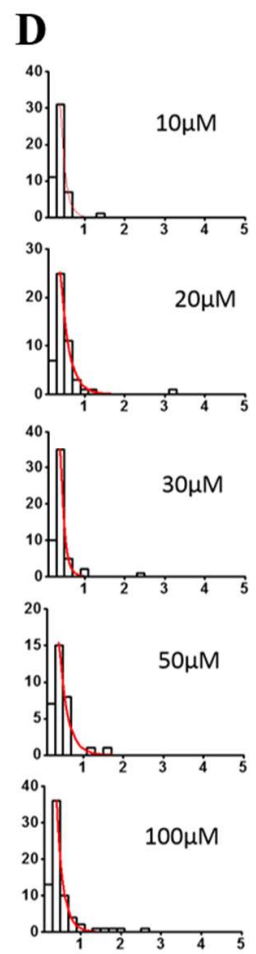

$t_{d u}$ (s)
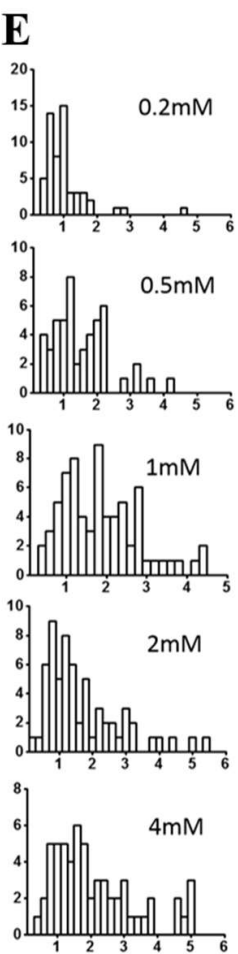

$t_{d c}$ (s)
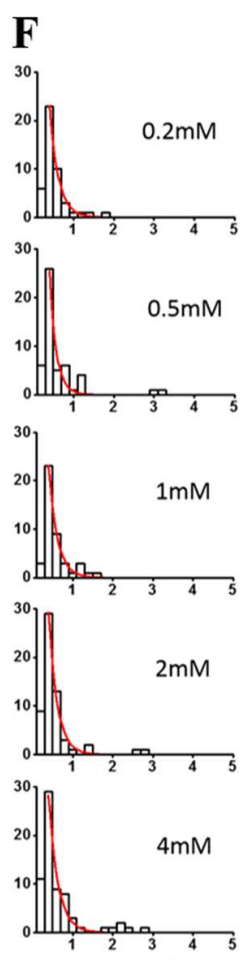

$t_{d u}(s)$

Figure S13. Effects of vanadate and ADP on remodeling kinetics. Representative

fluorescence intensity time traces showing remodeling events in the presence of $1 \mathrm{mM}$ ATP and $100 \mu \mathrm{M}$ vanadate (A), or in the presence of $100 \mathrm{mM}$ ATP and $4 \mathrm{mM}$ ADP (B). Vanadate titration of $(C) t_{d c}$, and (D) $t_{d u}$ for the nucleosome exit side at $1 \mathrm{mM} \mathrm{ATP.} t_{d u}$ histograms were fitted to single-exponential functions (red lines). ADP titration of (E) $t_{d c}$, and $(F) t_{d u}$ for the nucleosome exit side at $100 \mu \mathrm{M}$ ATP. $t_{d u}$ histograms were fitted to single-exponential functions (red lines). 
A
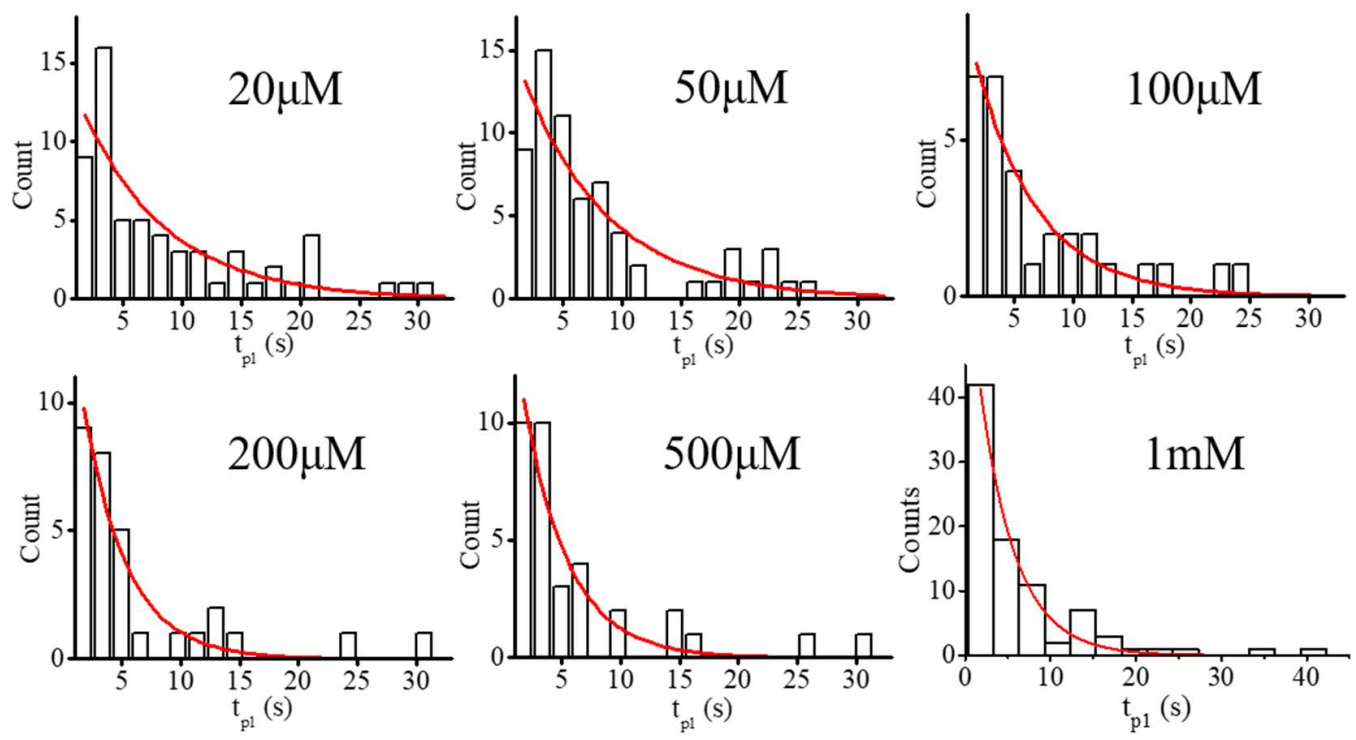

B

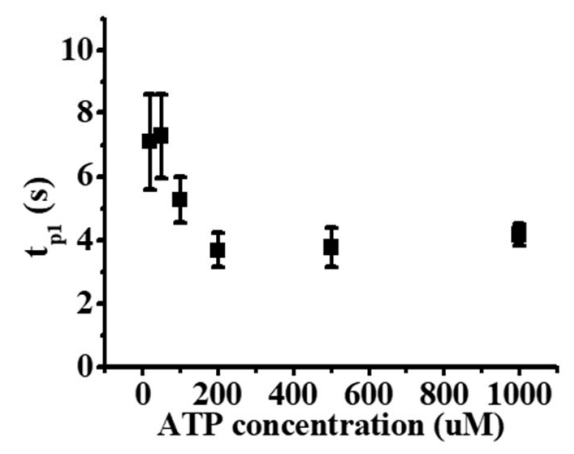

Figure S14. ATP dependence of $t_{p 1}$. (A) Histograms of $t_{p 1}$ at varying ATP concentration. (B) ATP titration of $t_{p 1}$. The data were obtained by fitting histograms to single-exponential functions (red lines in $(\mathrm{A})$ ). 
A

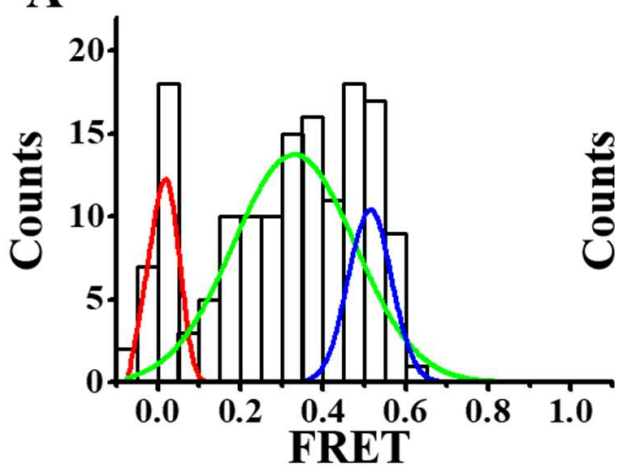

C

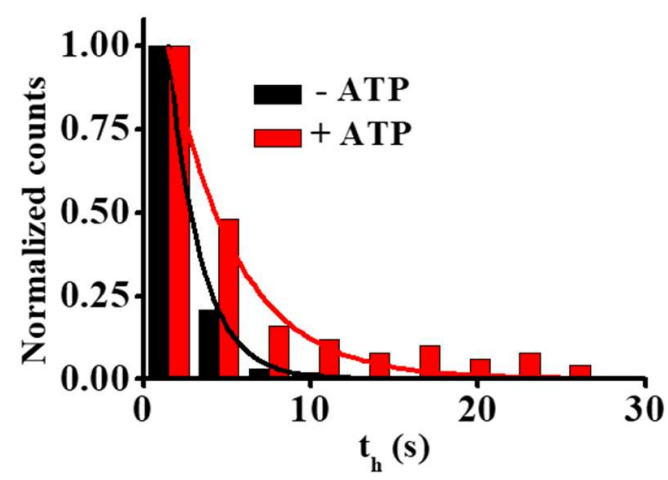

D

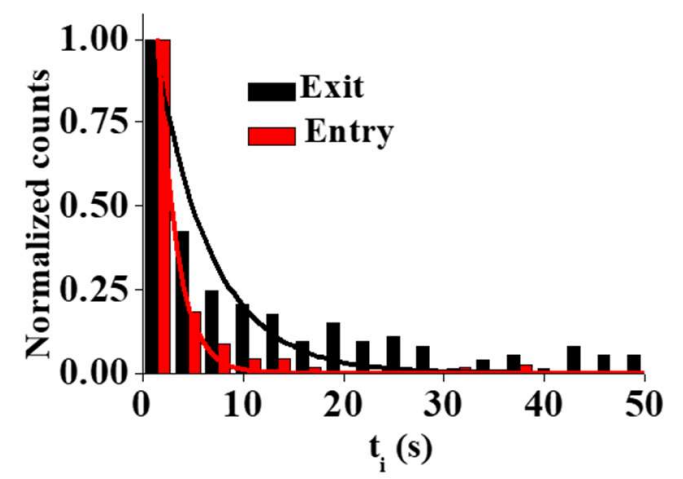

Figure S15. Supplementary data to characterize the entry-side remodeling. (A)

FRET histogram of the entry-side nucleosome before remodeling. Like the exit-side nucleosome, three FRET peaks were observed. Only the high FRET population was used for further analysis. (B) FRET histograms after first remodeling. As with the exit-side nucleosome, two distinct FRET distributions were observed. (C) High FRET dwell time $\left(\mathrm{t}_{\mathrm{h}}\right)$ on the entry side with (red) and without ATP (black), and their fit to singleexponential functions (solid lines). (D) Comparison of the initiation time $\left(\mathrm{t}_{\mathrm{i}}\right)$ of the entryside (red) and the exit-side nucleosome (black), and their fit to single-exponential functions (solid lines). 


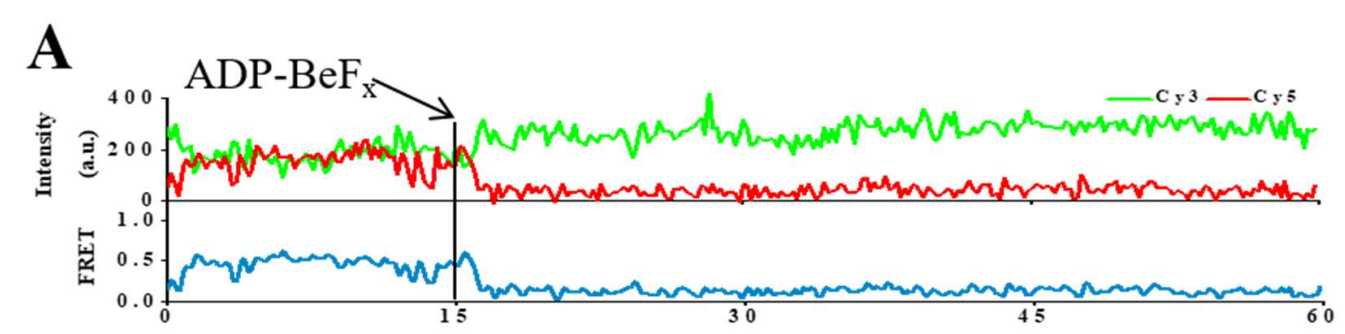

B

Time (s)

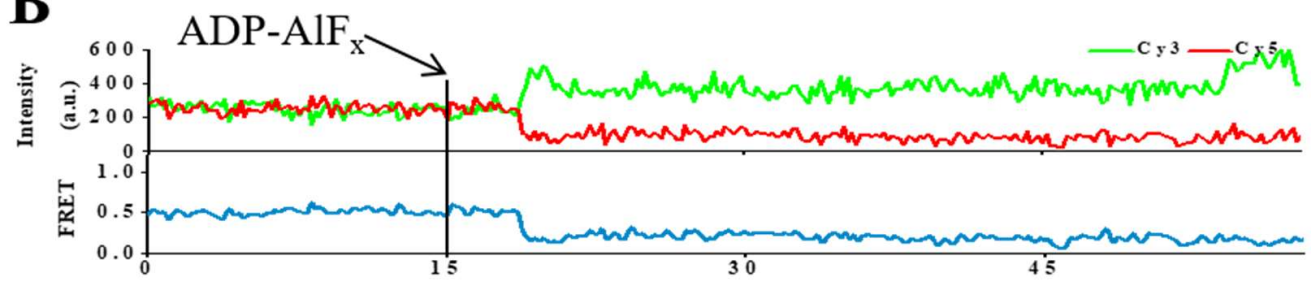

Time (s)

C

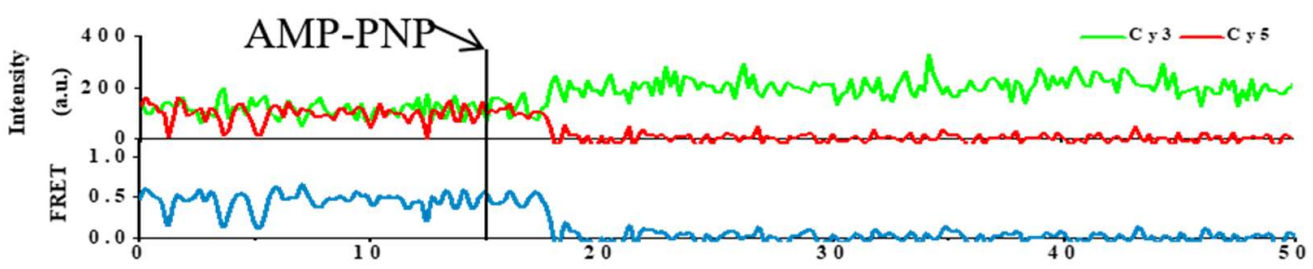

Time (s)

Figure S16. FRET down spikes on the entry side in the presence of various ATP analogs. Stable FRET down spikes were observed in the presence of (A) 1mM ADP-

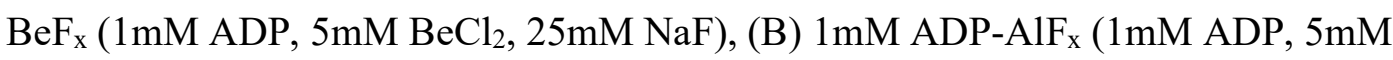
$\mathrm{AlCl}_{3}, 25 \mathrm{mM} \mathrm{NaF}$ ), or (C) 1mM AMP-PNP. 


\begin{tabular}{|cccc|}
\hline & SHL & +7 & +6
\end{tabular}

Figure S17. DNA sequences used to study the correlation between DNA unwrapping on the exit side and nucleosome remodeling. 